\title{
Constraints on Automorphic Forms of Higher Derivative Terms from Compactification
}

\author{
Finn Gubay, Neil Lambert and Peter West \\ Department of Mathematics \\ King's College London \\ The Strand, London \\ WC2R 2LS, UK
}

finn.gubay@kcl.ac.uk, neil.lambert@kcl.ac.uk, peter.west@kcl.ac.uk

\begin{abstract}
By dimensionally reducing the higher derivative corrections of ten-dimensional IIB theory on a torus we deduce constraints on the $E_{n+1}$ automorphic forms that occur in $d=10-n$ dimensions. In particular we argue that these automorphic forms involve the representation of $E_{n+1}$ with fundamental weight $\vec{\lambda}^{n+1}$, which is also the representation to which the string charges in $d$ dimensions belong. We also consider a similar calculation for the reduction of higher derivative terms in eleven-dimensional M-theory.
\end{abstract}




\section{Introduction}

It is well-known that the type IIA [1-3] and type IIB [4-6] supergravity theories in 10-dimensions are the low energy effective actions (at second order in derivatives) of the type II string theories. Furthermore Eleven-dimensional supergravity [7] is the low energy effective action of one of the limits of M-theory. Upon dimensional reduction on a torus to $d=10-n$ dimensions all these theories become equivalent and furthermore posses a large and unexpected duality symmetry $E_{n+1}[8-11]$

These supergravity theories are important as they encode all perturbative and nonpertubative effects, many of which cannot yet be calculated from first principles in String Theory or M-theory. The higher derivative terms of the effective action also encode much more structure of the fundamental theory and their study is of considerable interest. Since brane charges must be quantised $[12,13]$ and are rotated into each other under $E_{n+1}$, only a discrete subgroup of $E_{n+1}$ can be preserved in the quantum theory. This led to conjectures of duality symmetries in four-dimensional String Theory $[14,15]$ and then unified into socalled U-duality [16] for all dimensions.

The study of U-duality groups and higher derivative terms was begun in [17] for the $R^{4}$ term of ten-dimensional type IIB string theory with U-duality group $S L(2, \mathbf{Z})$ and has been considerably extended to other terms [18-23]. Results also exist for higher derivative terms in less than ten dimensions [23-36]. The effective action consists of polynomial in the field strengths, Riemann curvatures, and derivatives of the scalar fields multiplied by functions of the scalar fields. These functions transform in a simple way under discrete $E_{n+1}$ transformations and can be identified with non-holomorphic automorphic forms. In fact in most terms considered so far the functions transform trivially.

The theory of automorphic forms is complicated and still emerging. Large classes of these objects can be constructed by specifying a particular representation of a group $G$ along with a suitable subgroup $H$. In String Theory $G$ can be identified with the duality group $E_{n+1}$ and $H=I\left(E_{n+1}\right)$ with Cartan involution invariant subgroup (which is also the maximally compact subgroup) and the scalar fields are known to parameterise an $E_{n+1} / I\left(E_{n+1}\right)$ coset.

An important problem in String Theory is to identify the relevant automorphic forms that arise in various dimensions as coefficients of the higher derivative terms. One might hope that there are some relatively simple organising principles that control which automorphic forms appear. One of which is supersymmetry which relates various higher derivative terms of the same order in spacetime derivatives. Once a suitable automorphic form is known one can then in principle read off its perturbative and non-perturbative parts and hence deduce important information about the underlying quantum theory, such as non-renormalisation and instanton effects. These can then be checked against String 
Theory calculations [17-36] and non-renormalisation theorems [37].

One important question is: which representations enter into the construction of the automorphic forms? We will investigate this problem in this paper by examining the $E_{n+1}$ group structure of higher derivative terms in type IIB String theory and M-theory, after they have been dimensionally reduced to $d$ dimensions. We will see that this will enable us to deduce constraints on the representations that appear for general higher derivative terms in $d$-dimensions. Our results are very general and are consistent with conjectures and other results for special cases $[27,35,36]$.

This paper is a continuation of the analysis found in $[28,29]$. In particular we will dimensionally reduce a generic higher derivative term of type IIB String Theory on an $n$ torus to $d=10-n$ dimensions. When one does this one finds that each term in the $d$ dimensional effective action contains a factor of the form $e^{\sqrt{2} \vec{w} \cdot \vec{\phi}}$ for some vector $\vec{w}$. The fields $\vec{\phi}$ are the diagonal components of the metric, which encode the volume and other radii moduli of the torus, as well as any scalar fields in the higher dimensional theory such as the dilaton. Carrying out this for the supergravity theory, that is the lowest energy terms, we find that the vectors $\vec{w}$ that appear are the roots of $E_{n+1}$. Indeed, this provides the fastest way to see that there is very likely to be an $E_{n+1}$ symmetry of the lower dimensional theory. In references $[28,29]$ this was carried out for a generic higher derivative term of the effective action and one found not roots but weights of $E_{n+1}$. This in itself was evidence for an $E_{n+1}$ symmetry in the higher derivative corrections to string theory and the appearance of weights rather than roots was interpreted as evidence for automorphic forms as they involve weights of $E_{n+1}$. In this paper we take this work to its natural conclusion and compute the weights that should appear in the automorphic forms.

We carry out the calculation in a more streamlined manner than in references $[28,29]$ and generalise to any dimension $d \geq 3$. We assemble the fields of the lower dimensional theory, that occur with spacetime derivatives, into representations of the $d$-dimensional duality group $E_{n+1}$. We show that the higher derivative terms can be written as powers of the $E_{n+1}$ covariant field strengths along with additional factors of the dilaton and volume which are the remnants of the above $e^{\sqrt{2} \vec{w} \cdot \vec{\phi}}$ factors. We find that the left-over weight has a simple universal form for any term. For terms that arise at tree level in string perturbation in ten dimensions we find $\vec{w}=s \vec{\lambda}^{n+1}$, where $s=\left(l_{T}-2\right) / 4$ with $l_{T}$ counting the number of derivatives and $\vec{\lambda}^{n+1}$ the fundamental weight dual to $\vec{\alpha}_{n+1}$ (see Figure 1). The observation of [29] is that these additional factors must come from an automorphic form and therefore we are led to conclude that the automorphic form which multiplies a given higher derivative term involves the weight $\vec{\lambda}^{n+1}$. Moreover, for Eisenstein-like automorphic forms the leading order behaviour is given by $e^{-\sqrt{2} s \vec{\phi} \cdot \vec{\lambda}^{H}}$ ( for example see $[29,30]$ ), where $\vec{\lambda}^{H}$ is the highest weight of the representation used to construct the automorphic form. Thus our results suggest that the higher derivative terms always include an automorphic form built from a 
representation with highest weight $\vec{\lambda}^{n+1}$. We also perform a similar analysis in M-theory and see that the weight is (using the same labeling of the $E_{n+1}$ diagram), $\vec{w}=s \vec{\lambda}^{n-1}$ with $s=\left(l_{T}-2\right) / 6$. However it is important to note that we are in effect considering a particular limit and other representations could also appear but be subdominant in that limit.

The rest of this paper is organised as follows. In section 2 we perform our dimensional reduction analysis for type IIB string theory. In particular we perform a dimensional reduction with manifest $S L(2) \times S L(n)$ symmetry and show how this can be embedded into an $E_{n+1}$ symmetry. We include a detailed discussion of our methods as well as a description of $S L(2) \times S L(n)$ and $E_{n+1}$ coset constructions, including explicit examples. In section 3 we perform a similar analysis for the reduction of higher derivative terms of M-theory with a manifest $S L(n)$ symmetry. As mentioned above this leads to different weights of $E_{n+1}$ to that obtained in type IIB. In section 4 we conclude with a discussion of our results. We also include appendix A with a short review on the coset construction.

\section{Dimensional reduction of type IIB higher derivative terms}

In this section we will study the dimensional reduction of the higher derivative corrections of IIB string theory. Our methods follow those of section two of reference [28], suitably generalised for the reduction on an $n$ torus to any dimension rather than the three dimensions considered there. Our metric compactification ansatz is given by

$$
d \hat{s}^{2}=e^{2 \alpha \rho} d s^{2}+e^{2 \beta \rho} G_{i j}\left(d x^{i}+A_{\mu}^{i} d x^{\mu}\right)\left(d x^{j}+A_{\mu}^{j} d x^{\mu}\right)
$$

where $G_{i j}$ has unit determinant and

$$
\alpha=\sqrt{\frac{n}{2(D-2)(d-2)}}, \quad \beta=-\frac{(d-2) \alpha}{n} .
$$

These values of $\alpha$ and $\beta$ ensure that, provided one starts in $D$-dimensional Einstein frame, the resulting theory in $d$ dimensions is in Einstein frame with a standard normalisation for the kinetic term of the scalar $\rho$ which controls the volume of the torus. We have labeled the coordinates by $\left(x^{\mu}, x^{i}\right), \mu=0,1, \ldots, d-1 ; i=d, \ldots, D-1$. In the above equation $G_{i j}=e_{i}{ }^{\bar{k}} e_{j}{ }_{j} \delta_{\bar{k} \bar{l}}$ and $e_{i}{ }^{\bar{k}}$ is a vielbein but subject to det $e=1$. We adopt the convention that $i, j, k, \ldots$ are world indices and $\bar{i}, \bar{j}, \bar{k}, \ldots$ are tangent indices.

As is well-known, dimensionally reducing Einstein gravity on a torus leads to a theory that possesses an $S L(n, \mathbf{R})$ symmetry. In particular, the degrees of freedom of gravity associated with the torus, apart from the graviphotons, enter the lower dimensional theory through a non-linear realization of $S L(n, \mathbf{R})$ with local subgroup $S O(n)$. The latter is the 
Cartan involution invariant subgroup, i.e. $I(S L(n))=S O(n)$. This is to be expected as in the theory before the dimensional reduction the gravity degrees of freedom are in the non-linear realisation of $I S L(D)$ with local subgroup $S O(D)$.

The non-linear realisation is discussed for an arbitrary group in Appendix A. Using the local subgroup we can bring the $S L(n)$ group element to the form

$$
g_{s l_{n}}\left(\xi_{s l_{n}}\right)=e^{\sum_{\underline{\alpha}>0} E_{\underline{\alpha}} \chi_{\underline{\alpha}}} e^{-\frac{1}{\sqrt{2}} \underline{\phi} \cdot \underline{H}}
$$

where $\underline{H}$ forms the Cartan subalgebra, $E_{\underline{\alpha}}$ are positive root generators (when $\underline{\alpha}>0$ ) of $S L(n, \mathbf{R})$ respectively and $\xi_{s l_{n}}$ collectively denotes the fields $\chi_{\underline{\alpha}}$ and $\underline{\phi}$. In fact the terms which contain $g_{s l_{n}}\left(\xi_{s l_{n}}\right)$ alone are built out of the Cartan forms

$$
g_{s l_{n}}^{-1} \partial_{\mu} g_{s l_{n}}=P_{s l_{n} \mu}+Q_{s l_{n} \mu}
$$

where $P_{s l_{n} \mu}$ and $Q_{s l_{n} \mu}$ are symmetric and anti-symmetric in $\bar{i}$ and $\bar{j}$ respectively corresponding to the decomposition of the Cartan forms into those for $S O(n)$, i.e. $Q_{s l_{n} \mu}$, and its compliment.

In what follows we will construct the dimensionally reduced theory in such a way that its $S L(n, \mathbf{R})$ symmetry is manifest. To begin with we wish to find an expression for the inverse vielbein making use of the discussion of non-linear realisations (see appendix A). Let us denote the the fundamental highest weights of $S L(n)$ by $\underline{\lambda}^{i}$. The representation with highest weight $\underline{\lambda}^{1}$ corresponds to the vector representation, with a single lowered index. We denote the states of this representation by $|\psi\rangle=\psi_{i} \mid \underline{\mu}^{i}>$ where $\underline{\mu}^{i}$ are the weights in the root string of $\underline{\lambda}^{1}$, which we denote by $\left[\underline{\lambda}^{1}\right]$. From this linear representation we can construct the non-linearly transforming representation using equation (A.7) as follows

$$
\left|\varphi(\xi)>=\sum \varphi_{i}\right| \vec{\mu}^{i}>=L\left(g_{s l_{n}}^{-1}(\xi)\right)\left|\psi>=e^{\frac{1}{\sqrt{2}} \vec{\phi} \cdot \vec{H}} e^{\sum_{\vec{\alpha}>0}-\chi_{\vec{\alpha}} E_{\vec{\alpha}}}\right| \psi>
$$

and so we write

$$
\varphi_{i}=D\left(g_{s l_{n}}^{-1}\left(\xi_{s l_{n}}\right)\right)_{i}^{j} \psi_{j}
$$

Under an $S L(n)$ transformation this state transforms under a local $S O(n)$ and we may identify the change from $\psi_{i}$ to $\varphi_{i}$ as the familiar conversion from world to tangent indices using the inverse vielbein. The matrix element of $g_{s l_{n}}^{-1}$ in the vector representation is therefore given by

$$
\left(e^{-1}\right)_{i}^{j}=D\left(g_{s l_{n}}^{-1}\left(\xi_{s l_{n}}\right)\right)_{i}^{j}
$$

The right-hand end of equation $(2.5)$ contains the factor $e^{\frac{1}{\sqrt{2}} \phi \cdot\left[\underline{\lambda}^{1}\right]}$. Thus we find that the inverse vielbein $e_{i}{ }^{\bar{j}}$ contains factors of $e^{-\frac{1}{\sqrt{2}} \underline{\phi} \cdot\left[\underline{\lambda}^{1}\right]}$.

The dimensionally reduced theory will involve corrections that contain field strengths of the form $\mathcal{F}_{\mu_{1} \ldots \mu_{p} i_{1} \ldots i_{k}}$, where $i_{1}, \ldots i_{k}$ are worldvolume indices of the torus (there can 
also be $S L(2)$ indices which we address below). However, we can choose to work with tangent, rather than world, indices in the torus directions by using the inverse vielbein $\left(e^{-1}\right)_{i}^{j}$. Following the same argument we just used above, this can be viewed as the conversion of the linear rank $k$ antisymmetric representation of $S L(n, \mathbf{R})$ into a non-linear representation of $S L(n, \mathbf{R}) / S O(n)$ whose indices rotate under $S O(n)$. Thus we start from the linear representation $\sum_{i_{1}, \ldots i_{k}} F_{\mu_{1} \ldots \mu_{p} i_{1} \ldots i_{k}} \mid i_{1} \ldots i_{k}, \underline{\lambda}^{k}>$ and construct the non-linear realisation

$$
\sum_{i_{1}, \ldots i_{k}} \mathcal{F}_{\mu_{1} \ldots \mu_{p} \bar{i}_{1} \ldots \bar{i}_{k}}^{s l(n)}\left|i_{1} \ldots i_{k}, \underline{\lambda}^{k}>=L\left(g_{s l_{n}}^{-1}(\xi)\right) \sum_{i_{1}, \ldots i_{k}} F_{\mu_{1} \ldots \mu_{p} i_{1} \ldots i_{k}}\right| i_{1} \ldots i_{k}, \underline{\lambda}^{k}>
$$

We note that we have denoted the field strength with tangent indices by $\mathcal{F}_{\mu_{1} \ldots \mu_{p} \bar{i}_{1} \ldots \bar{i}_{k}}^{s l(n)}$. Its dependence on the metric of the torus is obtained by acting with $L\left(g_{s l_{n}}^{-1}(\xi)\right)$ on the states $\mid i_{1} \ldots i_{k}, \underline{\lambda}^{k}>$. Therefore one finds that the fields $\underline{\phi}$ associated with the Cartan subalgebra of $S L(n, \mathbf{R})$ occur in $\mathcal{F}_{\mu_{1} \ldots \mu_{p} \bar{i}_{1} \ldots \bar{i}_{k}}^{s l(n)}$ through the factor $e^{\frac{1}{\sqrt{2}} \phi \cdot\left[\lambda^{k}\right]}$.

\subsection{Review of $S L(2)$ Formulation of type IIB Supergravity}

We can treat the $S L(2, \mathbf{R})$ indices that arise in type IIB supergravity in a similar way. To illustrate this let us review in detail the $S L(2)$ invariant form of the ten-dimensional type IIB supergravity.

In ten dimensions the scalars belong to the non-linear realisation of $S L(2, \mathbf{R})$ with local subgroup $S O(2)$. The $S L(2, \mathbf{R})$ group element can be brought to the form

$$
g_{s l_{2}}(\tau)=e^{E \chi} e^{-\frac{1}{\sqrt{2}} \phi H}
$$

where $E$ and $H$ are the positive root and Cartan subalgebra generators of $S L(2, \mathbf{R})$ respectively. The scalars appear through the $S L(2, \mathbf{R})$ Cartan form

$$
g_{s l_{2}}^{-1} \partial_{\mu} g_{s l_{2}}(\tau)=P_{\mu}^{s l_{2}}+Q_{\mu}^{s l_{2}}=-\frac{1}{\sqrt{2}} \partial_{\mu} \phi H+\partial_{\mu} \chi e^{\phi} E
$$

Thus

$$
\begin{aligned}
P_{\mu}^{s l_{2}} & =-\frac{1}{\sqrt{2}} \partial_{\mu} \phi H+\partial_{\mu} \chi e^{\phi} \frac{(E+F)}{2} \equiv P_{\mu 1} \frac{H}{\sqrt{2}}+P_{\mu 2} \frac{(E+F)}{2}, \\
Q_{\mu}^{s l_{2}} & =\partial_{\mu} \chi e^{\phi} \frac{(E-F)}{2} .
\end{aligned}
$$

Under a local transformation $h=\exp \left(\theta \frac{(E-F)}{2}\right)$, the Cartan forms transform as $P_{\mu} \rightarrow$ $h P_{\mu} h^{-1}$ and so $P_{\mu 1} \rightarrow \cos \theta P_{\mu 1}+\sin \theta P_{\mu 2}, P_{\mu 2} \rightarrow \cos \theta P_{\mu 2}-\sin \theta P_{\mu 1}$. As a result we 
find that the complex quantity $P_{\mu}^{+} \equiv\left(P_{\mu 1}+i P_{\mu 2}\right)=-i e^{\phi} \partial_{\mu}\left(-\chi+i e^{-\phi}\right)$ transforms as $P_{\mu}^{+} \rightarrow e^{i \theta} P_{\mu}^{+}$. An invariant action for the scalars is then given by

$$
\begin{aligned}
S_{\text {scalar }} & =-\frac{1}{2} \int d^{10} x \operatorname{det} e P_{\mu}^{-} P^{+\mu} \\
& =-\frac{1}{2} \int d^{10} x \operatorname{det} e\left(\partial_{\mu} \phi \partial^{\mu} \phi+\partial_{\mu} \chi \partial^{\mu} \chi e^{2 \phi}\right) \\
& =-\frac{1}{2} \int d^{10} x \operatorname{det} e \frac{\left|\partial_{\mu} \tau\right|^{2}}{(\operatorname{Im} \tau)^{2}},
\end{aligned}
$$

where $P_{\mu}^{-}=\left(P_{\mu}^{+}\right)^{*}$ and in the last line we have introduced $\tau=-\chi+i e^{-\phi} ; \tau$ undergoes fractional linear transformations under the action of $S L(2, \mathbf{R})$ on this coset.

The IIB theory in ten dimensions contains a five-form field strength $F_{\mu_{1} \ldots \mu_{5}}$, which is a singlet under $S L(2, \mathbf{R})$. There are also two three-form field strengths $F_{\mu_{1} \mu_{2} \mu_{3} \alpha}=$ $3 \partial_{\left[\mu_{1}\right.} A_{\left.\mu_{2} \mu_{3}\right] \alpha}, \alpha=1,2$ which transform as the linear doublet representation of $S L(2, \mathbf{R})$. We can form the non-linear realisation by acting with $L\left(g_{s l_{2}}^{-1}\right)$, on this representation; we find, using equation (A.7), the corresponding non-linear representation;

$$
\mathcal{F}_{\mu_{1} \mu_{2} \mu_{3} \alpha}^{s l_{2}}\left|\alpha, \mu>=L\left(g_{s l_{2}}^{-1}(\tau)\right) F_{\mu_{1} \ldots \mu_{3} \alpha}\right| \alpha, \mu>,
$$

where $|1, \mu>=| \mu>$ and $|2, \mu>=|-\mu>$ with $\mu=\frac{1}{\sqrt{2}}$ being the fundamental representation of $S L(2, \mathbf{R})$. Viewing $\mathcal{F}_{\mu_{1} \mu_{2} \mu_{3} \alpha}^{s l_{2}}$ as a column vector we find that

$$
\mathcal{F}_{\mu_{1} \mu_{2} \mu_{3}}^{s l_{2}}=\left(\begin{array}{c}
e^{\frac{\phi}{2}}\left(F_{1 \mu \nu \rho}-\chi F_{2 \mu \nu \rho}\right) \\
e^{-\frac{\phi}{2}} F_{2 \mu \nu \rho}
\end{array}\right)
$$

which now only transform sunder the local subgroup $S O(2)$. Introducing the complex quantity $\mathcal{F}_{\mu \nu \rho}^{+}$this transformation becomes

$$
\mathcal{F}_{\mu \nu \rho}^{+} \equiv \mathcal{F}_{1 \mu \nu \rho}+i \mathcal{F}_{2 \mu \nu \rho}=e^{\frac{\phi}{2}}\left(F_{1 \mu \nu \rho}+\tau F_{2 \mu \nu \rho}\right) \rightarrow e^{i \theta} \mathcal{F}_{\mu \nu \rho}^{+}
$$

A manifestly $S L(2, \mathbf{R})$ invariant action is given by

$$
S_{2 f o r m}=\int d^{10} x \operatorname{det} e \mathcal{F}_{\mu_{1} \mu_{2} \mu_{3}}^{+} \mathcal{F}_{\nu_{1} \nu_{2} \nu_{3}}^{-}=\int d^{10} x \operatorname{det} e\left(e^{\phi}\left(F_{1}-\chi F_{2}\right)^{2}+e^{-\phi} F_{2}^{2}\right),
$$

where $\mathcal{F}_{\mu_{1} \mu_{2} \mu_{3}}^{-}=\left(F_{\mu_{1} \mu_{2} \mu_{3}}^{+}\right)^{*}$.

Including the standard Einstein-Hilbert term and a kinetic term for the 5-form field strength we arrive at the Bosonic part of the type IIB action

$$
S_{I I B}=\int d^{10} x \operatorname{det} e\left(R-P_{\mu}^{+} P^{\mu-}-\frac{1}{2 \cdot 3 !} \mathcal{F}_{\mu_{1} \mu_{2} \mu_{3}}^{+} \mathcal{F}^{\mu_{1} \mu_{2} \mu_{3}-}-\frac{1}{2 \cdot 5 !} \tilde{F}_{\mu_{1} \ldots \mu_{5}} \tilde{F}^{\mu_{1} \ldots \mu_{5}}\right) .
$$


Note that the five-form field strength equation of motion must be supplemented by a selfduality constraint and $\tilde{F}_{\mu_{1} \ldots \mu_{5}}=F_{\mu_{1} \ldots \mu_{5}}$ up to terms involving the 2-forms. Making the transition to string frame $e_{\mu}{ }^{\bar{\nu}}=e^{-\frac{\phi}{4}}\left(e_{S}\right)_{\mu}{ }^{\bar{\nu}}$, the type IIB effective action becomes

$$
\begin{aligned}
S_{I I B}=\int d^{10} x \operatorname{det}\left(e_{S}\right) & \left(e^{-2 \phi} R-\frac{1}{2} e^{-2 \phi} \partial_{\mu} \phi \partial^{\mu} \phi-\frac{1}{2} \partial_{\mu} \chi \partial^{\mu} \chi\right. \\
& \left.-\frac{1}{2 \cdot 3 !}\left(F_{1}-\chi F_{2}\right)^{2}-\frac{1}{2.3 !} e^{-2 \phi} F_{2}^{2}-\frac{1}{2 \cdot 5 !} \tilde{F}_{\mu_{1} \ldots \mu_{5}} \tilde{F}^{\mu_{1} \ldots \mu_{5}}\right) .
\end{aligned}
$$

We note from the factors of $e^{\phi}$ that occur that $F_{1 \mu_{1} \mu_{2} \mu_{3}}, P_{\mu 2}, \chi$ and $F_{\mu_{1} \ldots \mu_{5}}$ are in the R-R sector and $g_{\mu \nu}, \phi, P_{\mu 1}$ and $F_{2 \mu_{1} \mu_{2} \mu_{3}}$ in the NS-NS sector.

Let us now consider higher derivative terms. It will be useful for what follows to use a hat to denote a ten-dimensional quantity and suppress any spacetime indices. The higher derivative corrections in the IIB theory in ten dimensions can be written as a polynomial in the Riemann tensor $\hat{R}, \hat{P}^{s l_{2}}$, rank three field strength $\mathcal{F}_{3}^{ \pm}$and rank five field strength $F_{5}$ with coefficients that are $S L(2)$ automorphic forms. The generic term has the form

$$
\int d^{10} x \operatorname{det}(\hat{e}) \partial^{\hat{l}_{0}} \hat{R}^{\frac{\hat{l}_{R}}{2}}\left(\hat{P}_{\mu_{1} 1}^{s l_{2}}\right)^{\hat{l}_{1}}\left(\hat{P}_{\mu_{1} 2}^{s l_{2}}\right)^{\hat{l}_{1}^{\prime}}\left(\hat{\mathcal{F}}_{2 \mu_{1} \ldots \mu_{3}}\right)^{\hat{l}_{3}}\left(\hat{\mathcal{F}}_{1 \mu_{1} \ldots \mu_{3}}\right)^{\hat{l}_{3}^{\prime}}\left(\hat{F}_{\mu_{1} \ldots \mu_{5}}\right)^{\hat{l}_{5}^{\prime}} \hat{\Phi}_{s l_{2}}
$$

where $\hat{\Phi}_{s l_{2}}$ is a suitable automorphic form. As is well know the higher order corrections involve instantons and other solitonic objects and due to the quantisation conditions on the charges only the $S L(2, Z)$ part of the $S L(2, R)$ symmetry survives. The automorphic form depends on $\tau$ that is $\phi$ and $\chi$. We will be mainly interested in the $e^{\phi}$ dependence and we denote the leading dependence of $\hat{\Phi}_{s l 2}$ on $\phi$ by $\hat{\Phi}_{s l 2} \sim e^{-\hat{s} \phi}$.

It will be instructive to compute the $e^{\phi}$ dependence of the above ten dimensional higher derivative correction in string frame. The transition from Einstein frame to string frame is given by $\hat{e}=e^{-\frac{\phi}{4}} \hat{e}_{s}$. We find that the above term leads to the factor

$$
e^{\frac{\phi}{4}\left(\hat{l}_{0}+\hat{l}_{R}+\hat{l}_{1}+5 \hat{l}_{1}^{\prime}+\hat{l}_{3}+5 \hat{l}_{3}^{\prime}+5 \hat{l}_{5}^{\prime}-10-4 \hat{s}\right)} .
$$

Note that we have used a prime to denote contributions from R-R fields. At order $g$ in perturbation theory we have the contribution $e^{\phi(2 g-2)}$ and so we conclude that for a perturbative contribution

$$
\hat{s}=\frac{1}{4}\left(\hat{l}_{0}+\hat{l}_{R}+\hat{l}_{1}+5 \hat{l}_{1}^{\prime}+\hat{l}_{3}+5 \hat{l}_{3}^{\prime}+5 \hat{l}_{5}^{\prime}-2-8 g\right) .
$$

\subsection{Reduction of Higher Derivative Type IIB Terms}

In this paper we are interested in the dimensional reduction of ten dimensional higher derivative corrections of IIB string theory, that is terms as given in equation (2.19), on an 
$n$ torus to $d=10-n$ dimensions. As explained above, by working with the non-linear realisations we can formulate the result with a manifestly $S L(2) \otimes S L(n)$ symmetry. The resulting building blocks in $d$ dimensions are the Riemann tensor $R$ which is a $S L(2) \otimes$ $S L(n)$ singlet, the derivatives of the scalars which belong to the Cartan forms of $S L(2) \otimes$ $S L(n), P_{s l_{2} \otimes s l_{n}}$ and objects which are non-linear representations of $S L(2) \otimes S L(n)$. As mentioned above the latter arise if one works with "tangent space" quantities. These objects generically denoted by $\mathcal{F}$ are related to the usual field strengths, which transform linearly under $S L(2) \otimes S L(n)$, to the non-linearly realised objects $F$, by the generic equation

$$
\left|\mathcal{F}_{s l_{2} \otimes s l_{n}}>=L\left(g_{s l_{2} \otimes s l_{n}}^{-1}\right)\right| F>\equiv e^{\frac{1}{\sqrt{2}}(\underline{\phi} \cdot \underline{H}+\phi H)} e^{-\left(\sum_{\underline{\alpha}>0} E_{\underline{\alpha}} \chi_{\underline{\alpha}}+E \chi\right)} \mid F>.
$$

These $\mathcal{F}$ transform by field dependent $S O(n) \otimes S O(2)$ transformations and so it is easy to construct invariants using the Kronecker delta symbol. The $Q_{s l_{2} \otimes s l_{n}}$ component of the Cartan forms only enters when we find derivatives of the above objects where it plays the role of a connection.

We are particularly interested of the dependence in the dimensionally reduced action on the scalars $\phi, \rho$ and $\phi$ which we assemble into the $n+1$-vector

$$
\vec{\phi}=(\phi, \rho, \underline{\phi})
$$

The dependence on $\phi$ and $\underline{\phi}$, which are the Cartan fields associated with $S L(2) \otimes S L(n)$, occurs only inside the objects $\mathcal{F}_{s l_{2} \otimes s l_{n}}$. The exception is the $\phi$ dependence that arises from the ten dimensional automorphic form $\hat{\Phi}_{s l_{2}}$. The dependence on $\rho$ arises from the dimensional reduction of the vielbeins using the metric ansatz of equation (2.1) as was described in references $[28,29]$. The det $\hat{e}$ factor in the action leads to a factor of $e^{(d \alpha+n \beta) \rho}=$ $e^{2 \alpha \rho}$ while $\mathcal{F}_{s l_{2} \otimes s l_{n}} \mu_{1} \ldots \mu_{p} \bar{i}_{1} \ldots \bar{i}_{k}$ leads to the factor $e^{-\rho(p \alpha+k \beta)}$. To give a concrete example with $l$ factors of the latter field strength we find the generic term

$$
\int d^{10} x \operatorname{det} \hat{e}\left(\hat{\mathcal{F}}_{\mu_{1} \ldots \mu_{q}}^{s l_{2}}\right)^{l} \sim \sum_{p+k=q} \int d^{d} x \operatorname{det} e\left(\mathcal{F}_{s l_{2} \otimes s l_{n} \mu_{1} \ldots \mu_{p} \bar{i}_{1} \ldots \bar{i}_{k}}\right)^{l} e^{(2 \alpha-l(p \alpha+k \beta)) \rho} .
$$

The powers of $e^{\rho}$ associated with any other terms are also easily calculated.

The dimensional reduction of any term in the effective action of equation (2.17) leads to terms that contain the derivative of scalars, vierbein and gauge fields multiplied by factors of the form $e^{\sqrt{2} \vec{w} \cdot \vec{\phi}}$ for some $n+1$-vector $\vec{w}$ :

$$
\vec{w}=(w, \kappa, \underline{w})
$$

The first and third entries $w$ and $\underline{w}$ arise from the behaviour of the fields under the $S L(2, \mathbf{R}) \otimes S L(n)$ and can be read off from the action of $g_{s l_{2} \otimes s l_{n}}$ on the linearly realised 
representation using equation $(2.22)$. The second entry simply records the powers of $e^{\sqrt{2} \rho}$ that arise after dimensional reduction as just discussed.

For every factor of $F_{\mu_{1} \mu_{2} \mu_{3}}^{s l_{2}}$ that occurs one finds a corresponding factor of $e^{\frac{1}{\sqrt{2}} \phi[\mu]}$, where $[\mu]=\left\{\frac{1}{\sqrt{2}},-\frac{1}{\sqrt{2}}\right\}$ are the weights that appear in the fundamental representation of $S L(2, \mathbf{R})$. In particular the NS-NS and R-R field strengths come with the factors $e^{-\frac{\phi}{2}}$ and $e^{\frac{\phi}{2}}$ respectively as are most easily read off from equation (2.14).

In what follows it will be advantageous to also consider the dual version of certain fields. Let us consider a two-derivative term in the low energy effective action of the form

$$
\int d^{d} x \operatorname{det} e\left(\mathcal{F}_{s l_{2} \otimes s l_{n} \mu_{1} \ldots \mu_{p} \bar{i}_{1} \ldots \bar{i}_{k}}\right)^{2} e^{(2 \alpha-2(p \alpha+k \beta) \rho}
$$

where $\mathcal{F}_{s l_{2} \otimes s l_{n}}=g_{s l_{2} \otimes s l_{n}}^{-1} F, F=d A$. We can introduce the dual field strength $\mathcal{F}_{s l_{2} \otimes s l_{n} \mu_{1} \ldots \mu_{q} \bar{i}_{1} \ldots \bar{i}_{s}}^{D}$ defined by $\mathcal{F}_{s l_{2} \otimes s l_{n}}^{D}=g_{s l_{2} \otimes s l_{n}}^{-1} d A^{D}$ where $p+q=d$ and $k+s=n$. We then impose the Bianchi identity of $F=d A$ by adding to the action the term

$$
\begin{gathered}
\int d^{d} x \epsilon^{\mu_{1} \ldots \mu_{p} \nu_{1} \ldots \nu_{q}} \epsilon^{i_{1} \ldots \bar{i}_{k} j_{1} \ldots \bar{i}_{s}} F_{s l_{2} \otimes s l_{n} \mu_{1} \ldots \mu_{p} \bar{i}_{1} \ldots \bar{i}_{k}} F_{s l_{2} \otimes s l_{n} \nu_{1} \ldots \nu_{q} \bar{j}_{1} \ldots \bar{j}_{s}}^{D} \\
=\int d^{d} x \epsilon^{\mu_{1} \ldots \mu_{p} \nu_{1} \ldots \nu_{q}} \epsilon^{i_{1} \ldots \bar{i}_{k} j_{1} \ldots \bar{i}_{s}} \mathcal{F}_{s l_{2} \otimes s l_{n} \mu_{1} \ldots \mu_{p} \bar{i}_{1} \ldots \bar{i}_{k}} \mathcal{F}_{s l_{2} \otimes s l_{n} \nu_{1} \ldots \nu_{q} \bar{j}_{1} \ldots \bar{j}_{s}}^{D}
\end{gathered}
$$

where $F^{D}=d A^{D}$ and in the second line with have used the fact that $\operatorname{det}\left(g_{s l_{2} \otimes s l_{n}}\right)=1$. Note that if $\mathcal{F}_{s l_{2} \otimes s l_{n}}$ and $\mathcal{F}_{s l_{2} \otimes s l_{n}}^{D}$ have $S L(2)$ indices then an additional factor of $\epsilon^{a b}$ is needed in (2.27).

We can now view $\mathcal{F}_{s l_{2} \otimes s l_{n}}$ as an unconstrained field and integrate it out. Taking its equation of motion implies that

$$
\mathcal{F}_{s l_{2} \otimes s l_{n} \mu_{1} \ldots \mu_{p} \bar{i}_{1} \ldots \bar{i}_{k}} \sim \epsilon^{i_{1} \ldots \bar{i}_{k} j_{1} \ldots \bar{i}_{s}} \epsilon^{\mu_{1} \ldots \mu_{p} \nu_{1} \ldots \nu_{q}} \mathcal{F}_{s l_{2} \otimes s l_{n} \nu_{1} \ldots \nu_{q} \bar{j}_{1} \ldots \bar{j}_{s}}^{D} e^{2(-\alpha+(p \alpha+k \beta)) \rho}
$$

We will assume that we can use this lowest order dualisation equation in the higher order corrections. Therefore, for each factor of $\mathcal{F}_{s l_{2} \otimes s l_{n} \mu_{1} \ldots \mu_{p} \bar{i}_{1} \ldots \bar{i}_{k}}$ we find in the higher derivative terms

$$
\begin{aligned}
\mathcal{F}_{s l_{2} \otimes s l_{n} \mu_{1} \ldots \mu_{p} \bar{i}_{1} \ldots \bar{i}_{k}} e^{-(p \alpha+k \beta) \rho} & \sim \mathcal{F}_{s l_{2} \otimes s l_{n} \nu_{1} \ldots \nu_{q} \bar{j}_{1} \ldots \bar{j}_{s}}^{D} e^{-2 \alpha+(p \alpha+k \beta) \rho} \\
& \sim \mathcal{F}_{s l_{2} \otimes s l_{n} \nu_{1} \ldots \nu_{q} \bar{j}_{1} \ldots \bar{j}_{s}}^{D} e^{-(q \alpha+s \beta) \rho} .
\end{aligned}
$$

In the last step used equation (2.2). Hence, we get the same result if we use the original field or we use the dual field provided we take into account the correct number of indices. The reader may check this in specific cases including that of the graviphoton which first appears when reducing the Riemann tensor with a field strength that carries a single upper $i$ index. 
It is rather pleasing to compute the vectors $\vec{w}$ that arise when dimensionally reducing the IIB supergravity theory of equation (2.17) and show that one finds the weights of $E_{n+1}$.

\subsection{The $E_{n+1}$ symmetry in $d$ dimensions}

As discussed in the last section the dimensional reduction of the IIB theory including its higher derivative corrections, on an $n$ torus leads to a formulation in which the $S L(2) \otimes$ $S L(n)$ symmetry is manifest. However, the IIB supergravity theory when dimensionally reduced to $d=10-n$ dimensions actually possess an $E_{n+1}$ symmetry, of which a discrete subgroup is preserved in the quantum theory. Evidence for this conjecture has been obtain in a variety of works such as [17-36]. The Dynkin diagram of $E_{n+1}$ suited to the IIB theory is given by

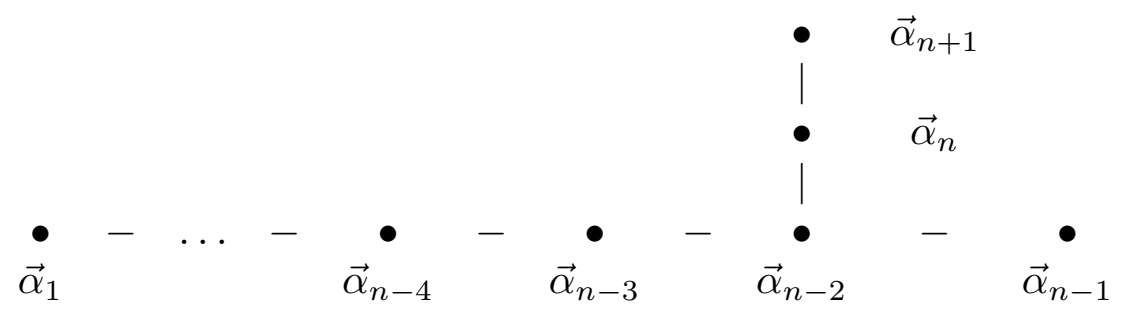

Fig 1: Dynkin diagram for $E_{n+1}$ in type IIB labelling

The relevant $S L(2) \otimes S L(n)$ subalgebra of $E_{n+1}$ is found by deleting the node labeled $n$ in the Dynkin diagram of Figure 1. The $S L(2)$ factor is just the $S L(2)$ symmetry of the IIB supergravity theory and arises from the node labeled $n+1$, while the $S L(n)$ symmetry is part of the gravity symmetry of the ten dimensional theory that now belongs to the torus and corresponds to the nodes labeled 1 to $n-1$.

These features are particularly apparent when one considers the $E_{11}$ formulation of the IIB theory $[38,39]$. The $E_{n+1}$ Dynkin diagram emerges from the $E_{11}$ Dynkin diagram, given just below, by deleting the node $d$ to find the algebra $S L(d) \otimes E_{n+1}$.

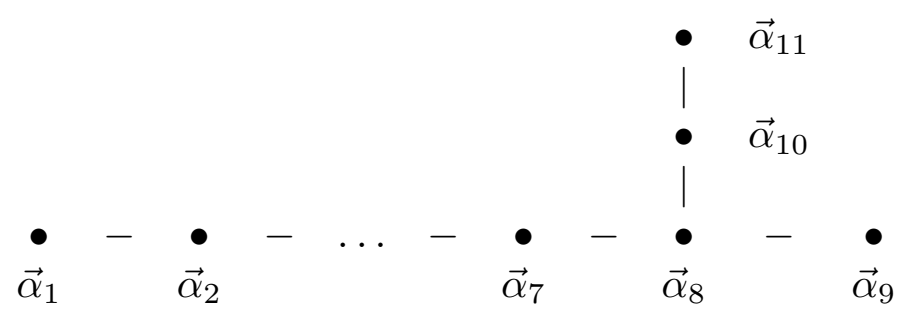

Fig 2: Dynkin diagram for $E_{11}$

The nodes labeled 1 to 9 of the $E_{11}$ Dynkin diagram are called the gravity line as they are associated with ten dimensional gravity. After the deletion of the node $d$, this 
line gives rise to $S L(d) \otimes S L(n)$ which is associated with gravity in $d$ dimensions and the $S L(n)$ of the now internal $E_{n+1}$ symmetry.

As already mentioned if one computes the weights $\vec{w}$ that arise from the dimensional reduction of the IIB supergravity theory using the techniques given in the last section one readily finds that they are the weights of $E_{n+1}$. While this is a strong indication of an underlying $E_{n+1}$ symmetry the detailed dimensional reduction is required to prove the existence of this symmetry in $d$ dimensions. In this process one finds that the $S L(2) \otimes S L(n)$ representations that the fields belong to collect up to form a representation of $E_{n+1}$. In this paper it will be essential to understand how the representations of $E_{n+1}$ that occur decompose into representations of $S L(2) \otimes S L(n)$ as this will allow us to compare the $E_{n+1}$ formulation of the higher derivative corrections with that arising from dimensional reduction from ten dimensions. It is from this comparison that we will be able to deduce some properties of the automorphic form in $d$ dimensions. The review [40] on U-duality discusses $E_{n+1}$ representations but here we will need the explicit form for the weights.

\begin{tabular}{|c|c|c|c|c|c|c|}
\hline$d$ & $E_{n+1}$ & $I\left(E_{n+1}\right)$ & $F_{2}$ & $F_{3}$ & $F_{4}$ & $F_{5}$ \\
\hline 10 & $S L(2)$ & $S O(2)$ & & $\mathbf{2}$ & & $\mathbf{1}$ \\
8 & $S L(3) \times S L(2)$ & $S O(3) \times S O(2)$ & $(\overline{\mathbf{3}}, \mathbf{2})$ & $(\overline{\mathbf{3}}, \mathbf{1})$ & $(\mathbf{1}, \mathbf{2})$ & \\
7 & $S L(5)$ & $S O(5)$ & $\overline{\mathbf{1 0}}$ & $\mathbf{5}$ & $\overline{\mathbf{5}}$ & \\
6 & $S O(5,5)$ & $S O(5) \times S O(5)$ & $\mathbf{1 6}$ & $\mathbf{1 0}$ & & \\
5 & $E_{6}$ & $U S P(8)$ & $\mathbf{2 7}$ & & & \\
4 & $E_{7}$ & $S U(8)$ & $\mathbf{5 6}$ & & & \\
3 & $E_{8}$ & $S O(16)$ & & & & \\
\hline
\end{tabular}

Table 1: $E_{n+1}, I\left(E_{n+1}\right)$ and representation of the Field Strengths

The scalars, denoted $\xi_{E}$, in $d$ dimensions belong to a non-linear realisation of $E_{n+1}$ with local subgroup $I\left(E_{n+1}\right)$ where $I(G)$ denotes the Cartan involution invariant subgroup of $G$. These local subgroups are given in Table 1. Following the discussion of non-linear realisations given in appendix A we find the transformations of equation (A.6). Given a group element $g_{E}\left(\xi_{E}\right)$ of $E_{n+1}$ we can use the local transformation $I\left(E_{n+1}\right)$ to cast it in the form

$$
g_{E}\left(\xi_{E}\right)=e^{\sum_{\vec{\alpha}>0} E_{\vec{\alpha}} \chi_{\vec{\alpha}}} e^{-\frac{1}{\sqrt{2}} \vec{\phi} \cdot \vec{H}}
$$

where $E_{\vec{\alpha}}$ are the positive root and $\vec{H}$ the Cartan subalgebra generators of $E_{n+1}$. The fields $\vec{\phi}$ and $\chi_{\vec{\alpha}}$ are the scalar fields of the theory which we have denoted collectively by $\xi_{E}$. The dynamics of the scalars are constructed, as usual, out of the Cartan form $g_{E}^{-1} d g_{E}=P_{E}+Q_{E}$, where $Q_{E}$ lies in the Lie-algebra of $I\left(E_{n+1}\right)$. 
The gauge fields transforms as linear representations of $E_{n+1}$; their representations are given in Table 1 . Note that care must be taken for $d / 2$-form field strengths as these generally only fill out $E_{n+1}$ representations if their electromagnetic duals are also included. However, it is desirable to use the scalar fields $\xi_{E}$ to convert the fields strengths $F_{E}$ which belong to linear realisations of $E_{n+1}$, into tensors denoted $\mathcal{F}_{\mathcal{E}}$ which transform non-linearly under $E_{n+1}$, using equation (A.8). We may write the relation in the generic form

$$
\left|\mathcal{F}_{E}>=L\left(g_{E}^{-1}\left(\xi_{E}\right)\right)\right| F_{E}>
$$

Under an $E_{n+1}$ transformation these change as

$$
\left|\mathcal{F}_{E}>\rightarrow L\left(h^{-1}\right)\right| \mathcal{F}_{E}>
$$

where $h \in I\left(E_{n+1}\right)$. We can write $g_{E}\left(\xi_{E}\right)=g_{s l_{2} \otimes s l_{n}}\left(\xi_{s l_{2} \otimes s l_{n}}\right) g^{\prime}$ where $g^{\prime}$ contains the Cartan and positive root generators of $E_{n+1}$ which are outside $S L(2) \otimes S L(n)$. Therefore we can write

$$
\begin{aligned}
\mid \mathcal{F}_{E}> & =L\left(g_{E}^{-1}\left(\xi_{E}\right)\right) \mid F> \\
& =L\left(\left(g^{\prime}\right)^{-1} L\left(g_{s l_{2} \otimes s l_{n}}^{-1}\right)\left(\xi_{s l_{2} \otimes s l_{n}}\right) \mid F>\right. \\
& =\sum_{(\mu, \underline{\lambda})} L\left(\left(g^{\prime}\right)^{-1}\right) \mid \mathcal{F}_{s l_{2} \otimes s l_{n}}^{(\mu, \underline{\lambda})}>
\end{aligned}
$$

Hence the $E_{n+1}$ non-linear realisations $\mathcal{F}_{E}$ that appear in the $E_{n+1}$ formulation of the theory can be written as $L\left(\left(g^{\prime}\right)^{-1}\right)$ acting on the non-linear realisations $\mathcal{F}_{s l_{2}}^{(\mu, \underline{\lambda})} s_{n}$. The superscript $(\mu, \underline{\lambda})$ are the highest weights of the different $S L(2) \otimes S L(n)$ representations that arise in the decomposition of the linear representation $F$, that is $F=\sum_{(\mu, \underline{\lambda})} F_{s l_{2}}^{(\mu, \underline{\lambda})} s l_{n}$.

We will primarily be interested in the scalar fields associated with the Cartan subalgebra of $E_{n+1}$. The subalgebra $S L(n) \otimes S L(2)$ has $n$ such fields $\underline{\phi}$ and $\phi$ which are associated with the nodes $1 \ldots, n-1$ and node $n+1$ of the $E_{n+1}$ Dynkin diagram respectively. The remaining Cartan field in $E_{n+1}$ is $\rho$ and this is associated with the deleted noded, that is the node $n$. Restricting $g$ to the Cartan sub-algebra, denoted $g_{c}=e^{-\frac{1}{\sqrt{2}} \vec{\phi} \cdot \vec{H}}$ we find that

$$
\begin{aligned}
L\left(g_{c}^{-1}\right) \mid \vec{\lambda}> & =e^{+\frac{1}{\sqrt{2}} \vec{\lambda} \cdot \vec{\phi}} \mid \vec{\lambda}> \\
& =e^{\frac{1}{\sqrt{2}}(\vec{\lambda})_{n} \rho} e^{+\frac{1}{\sqrt{2}} \underline{\lambda} \cdot \underline{\phi}} e^{+\frac{1}{\sqrt{2}}(\vec{\lambda})_{n+1} \phi} \mid \vec{\lambda}> \\
& =e^{\frac{1}{\sqrt{2}}(\vec{\lambda})_{n} \rho} L\left(g_{s l_{2} \otimes s l_{n} c}^{-1}\right) \mid \vec{\lambda}>
\end{aligned}
$$

when acting on a state in a representation of $E_{n+1}$ with weight $\vec{\lambda}$. Here $(\vec{\lambda})_{n}$ is the nth component of $\vec{\lambda}$. We are interested in comparing the $E_{n+1}$ formulation of the higher derivative corrections in $d$ dimensions with those obtained by dimensional reduction from 
ten dimensions, both of which can be written in terms of non-linear realisation of $S L(2) \otimes$ $S L(n)$ symmetry, i.e. in terms of $\mathcal{F}_{s l_{2}}^{(\mu, \lambda)} \dot{\otimes} l_{n}$. Consequently, it is the difference which is of most interest, namely the $e^{-\frac{1}{\sqrt{2}}(\vec{\lambda})_{n} \rho}$ factors. In the $E_{n+1}$ formulation these arise by decomposing the $E_{n+1}$ building blocks $\mathcal{F}$ as in equation (2.31) and then using equation (2.32) while in the dimensional reduction they arise from the metric ansatz of equation (2.1).

We assume that the higher derivative corrections to the IIB theory are invariant under a discrete $E_{n+1}$ symmetry. The fields transform in the same way as for the IIB supergravity theory in $d$ dimensions, but under the discrete group. The terms in the $d$ dimensional effective action will be of the generic form

$$
\int d^{d} x \operatorname{det} e \partial^{l_{0}} R^{\frac{l_{R}}{2}} P_{E}^{l_{1}}\left(\mathcal{F}_{E \mu_{1}}\right)^{l_{1}}\left(\mathcal{F}_{E \mu_{1} \mu_{2}}\right)^{l_{2}} \Phi_{E} \ldots
$$

where $\mathcal{F}_{E \mu_{1}}, \ldots$ are the $E_{n+1}$ non-linear realisations constructed in equation (2.31) and $\Phi_{E}$ is function of the scalars $\xi$ which transforms under the discrete symmetry as

$$
\Phi_{E} \rightarrow D\left(h^{-1}\right) \Phi_{E},
$$

for $h \in I\left(E_{n+1}\right)$ and $D(h)$ being in the representation that $\Phi_{E}$ belongs to. However, $\Phi_{E}$ has a non-holomorphic dependence on the scalars and we will refer to it as a non-holomorphic automorphic form.

A formulation of automorphic forms which transform as in equation (2.34) was given in reference [29]. To construct such a non-holomorphic automorphic form for a discrete group $G$ one chooses a linear representation of $G$ denoted $\mid \psi>$ and considers $\left|\varphi>=L\left(g^{-1}\right)\right| \psi>$ where $g(\xi)$ is an element of $G$ that is subject to the transformations of equation (A.2), that is it is a non-linear realisation and $|\varphi\rangle$ is the non-linear realisation constructed from $|\psi\rangle$ using equation (A.8). The automorphic form is a suitable function of $\varphi$. The simplest case is that of a scalar automorphic form that is given by

$$
\Phi=\sum_{\mid \psi>\neq 0} \frac{1}{<\varphi|\varphi\rangle^{s}} .
$$

For our case $G=E_{n+1}$ and $\xi$ are the scalar fields of the theory, include those associated with Cartan subalgebra which we have labeled by $\vec{\phi}=(\phi, \rho, \phi)$. To leading order the automorphic form will have a dependence on these scalars which we denote by

$$
\Phi_{E} \sim e^{-\sqrt{2} \vec{\lambda}_{\Phi} \cdot \vec{\phi}}
$$

where $\vec{\lambda}_{\Phi}$ a weight of the representation. For the automorphic form of equation (2.35) $\Phi \sim e^{-\sqrt{2} s \vec{\lambda}_{H} \cdot \vec{\phi}}$ where $\vec{\lambda}_{H}$ is the highest weight of the represenation used to build the automorphic form. 
In this paper we will want to compare the terms in the effective action of equation (2.33) in their $E_{n+1}$ formulation with those obtained from the dimensional reduction of the higher derivative terms in ten dimensions given in equation (2.19). This will allow us to place restrictions on the automorphic form $\Phi_{E}$ in $d$ dimensions and in particular the weights $\lambda^{\phi}$ that can appear in it. For almost all terms this will require the decomposition of the $E_{n+1}$ representations that occur into $S L(2) \otimes S L(n)$ representations.

The simplest examples are terms in the effective action of equation (2.33) that only involve powers of the the Riemann tensor in $d$ dimensions since the Riemann tensor is a singlet of $E_{n+1}$. This contribution comes from the dimensional reduction of the similar term in ten dimensions, namely that of equation (2.19) with only $\hat{l}_{R}=l_{R}$ non vanishing. Since the Riemann tensor, in tangent frame, possess two powers of the inverse vierbein we find a factor of $e^{-2 \alpha \rho}$ for each Riemann tensor and a factor of $e^{2 \alpha \rho}$ from det $\hat{e}$. From the automorphic form in ten dimensions we find, at leading order, a factor of $e^{-\hat{s} \phi}$. Thus from dimensional reduction we find in $d$ dimensions the term

$$
\int d^{d} x \operatorname{det} e R^{\frac{l_{R}}{2}} \Phi_{E} e^{-\hat{s} \phi-\left(l_{R}-2\right) \alpha \rho} .
$$

Comparing this with the $E_{n+1}$ formulation in $d$ dimensions which is of the form $\int d^{d} x \operatorname{det} e R^{\frac{l_{R}}{2}} \hat{\Phi}_{E}$ we see that the additional factor of $\phi$ and $\rho$ must arise from the automorphic form $\Phi_{E}$ and so we find that,

$$
\vec{\lambda}_{\Phi}=\left(\frac{\hat{s}}{\sqrt{2}}, \alpha \frac{\left(l_{R}-2\right)}{\sqrt{2}}, \underline{0}\right)
$$

From equation $(2.21)$ we have $\hat{s}=\frac{1}{4}\left(l_{R}-2-8 g\right)$ and taking the leading contribution at $g=0$ we conclude that $\vec{\lambda}_{\Phi}=\frac{1}{4}\left(l_{R}-2\right) \vec{\lambda}^{n+1}$ where $\lambda^{n+1}=\left(\frac{1}{\sqrt{2}}, \frac{1}{2 x}, \underline{0}\right)$ and we have used the relation $x^{-1}=4 \sqrt{2} \alpha$. Thus the automorphic form has the leading order behaviour $\Phi \sim e^{-\sqrt{2} \frac{1}{4}\left(l_{R}-2\right) \vec{\lambda}^{n+1} \cdot \vec{\phi}}$. Hence for terms which contain only the Riemann curvature it is straight forward to to compute the leading behaviour of the automorphic form. In what follows we will carry out this calculation for all possible terms, but as we will see this involves some much more sophisticated group theory.

In order to study the remaining terms. We consider the possible building blocks that arise in the dimensional reduction from ten dimensions and compare these with those in the $E_{n+1}$ formulation. As we have explained above the latter can be expressed in terms of non-linear realisations of $S L(2) \otimes S L(n)$ which agree with the same objects found from dimensional reduction. The difference arises from the $\rho$ dependence. To find this difference we must decompose the representations of $E_{n+1}$ into those of $S L(2) \otimes S L(n)$. We do this following the techniques [41-43] developed for the study of the $E_{11}$ symmetry. As mentioned above, deleting the node $n$ in the Dynkin diagram of $E_{n+1}$ results in the 
algebras $S L(2) \otimes S L(n)$. We may write the simple roots of $E_{n+1}$ as

$$
\vec{\alpha}_{n+1}=\left(\beta_{1}, 0,0\right), \vec{\alpha}_{n}=(0, x, \underline{0})-\vec{\nu}, \vec{\alpha}_{i}=\left(0,0, \underline{\alpha}_{i}\right), i=1, \ldots, n-1
$$

where $\vec{\nu}=\left(\mu_{1}, 0, \underline{0}\right)+\left(0,0, \underline{\lambda}^{n-2}\right)$. Also, the $\underline{\alpha}_{i}$ and $\underline{\lambda}^{i}$ are the simple roots and fundamental weights of $S L(n)$ and $\beta_{1}=\sqrt{2}$ and $\mu=\frac{1}{\sqrt{2}}$ the simple root and fundamental weight of $S L(2)$. Demanding that $\vec{\alpha}_{n}^{2}=2$ we find that $x=\sqrt{\frac{8-n}{2 n}}=(4 \sqrt{2} \alpha)^{-1}$.

The fundamental weights of $E_{n+1}$, denoted $\lambda^{a}, a=1, \ldots, n+1$, satisfy $\alpha_{a} \cdot \lambda^{b}=\delta_{a, b}$ and are given by

$$
\vec{\lambda}^{i}=\left(0, \frac{1}{x} \underline{\lambda}^{n-2} \cdot \underline{\lambda}^{i}, \underline{\lambda}^{i}\right), \quad \vec{\lambda}^{n}=\left(0, \frac{1}{x}, \underline{0}\right), \quad \vec{\lambda}^{n+1}=\left(\mu, \frac{1}{2 x}, \underline{0}\right) .
$$

Any root of $E_{n+1}$ can be written as

$$
\vec{\alpha}=n_{c} \vec{\alpha}_{n}+m \vec{\beta}_{1}+\sum_{i} n_{i} \vec{\alpha}_{i}=n_{c}(0, x, 0)-\vec{\lambda}
$$

where $\vec{\lambda}=n_{c} \vec{\nu}-\sum_{i} n_{i}\left(0,0, \underline{\alpha}_{i}\right)-m\left(\beta_{1}, 0, \underline{0}\right)$. The latter is a weight of $S L(2) \otimes S L(n)$. If a representation of $S L(2) \otimes S L(n)$ occurs in the decomposition of the adjoint representation of $E_{n+1}$ its highest weight must occur as one of the $\lambda$ 's for some positive integers $m, n_{i}$ and $n_{c}$. We refer to the integer $n_{c}$ as the level and we can analyse the occurrence of highest weights level by level using the techniques of references [41-43]. Clearly, at level zero i.e $n_{c}=0$ we have just the adjoint representation of $S L(2) \otimes S L(n)$. The result is that the adjoint representation of $E_{n+1}$ contains the adjoint representation of $S L(2) \otimes S L(n)$ at $n_{c}=0$ together with the following highest weight representations of $S L(2) \otimes S L(n)$

$$
\begin{array}{cccc}
n_{c}=1 & n_{c}=2 & n_{c}=3 & n_{c}=4 \\
\left(\mu, \underline{\lambda}^{2}\right) & \left(0, \underline{\lambda}^{4}\right) & \left(\mu, \underline{\lambda}^{6}\right) & \left(0, \underline{\lambda}^{1}+\underline{\lambda}^{n-7}\right)
\end{array} .
$$

Thus the weights in the adjoint representation of $E_{n+1}$ then have the from

$$
\begin{gathered}
\left(\left[\beta_{1}\right], 0, \underline{0}\right),\left(0,0,\left[\underline{\alpha}_{1}+\ldots+\underline{\alpha}_{n_{1}}\right]\right),\left(\left[\mu_{1}\right], x,\left[\underline{\lambda}^{1}\right]\right), \\
\left(0,2 x,\left[\underline{\lambda}^{4}\right]\right),\left(\left[\mu_{1}\right], 3 x,\left[\underline{\lambda}^{6}\right]\right),\left(0,4 x,\left[\underline{\lambda}^{1}+\underline{\lambda}^{n-7}\right]\right) .
\end{gathered}
$$

These correspond to the adjoint of $S L(2) \otimes S L(n)$ at $n_{c}=0$ as well as the generators

$$
\begin{array}{cccc}
n_{c}=1 & n_{c}=2 & n_{c}=3 & n_{c}=4 \\
R^{\alpha i j} & R^{i_{1} \ldots i_{4}} & R^{\alpha, i_{1} \ldots i_{6}} & R^{j_{1} \ldots j_{7} i}
\end{array}
$$

The maximum value of $n_{c}$ that contributes is $n_{c}=1,2,2,3,4$ for $n=3,4,5,6,7$ respectively as is clear from the index structures of the generators. The reader may verify that once 
the additional negative root generators are included this collection of generators has the correct count of generators for $E_{n+1}$ for $n=3, \ldots, 7$.

The Cartan forms of $E_{n+1}$ belong to the adjoint representation and so using equation (2.43) we find that the coset component $P_{E a}$ decomposes into the Cartan forms $P_{s l_{n} \otimes s l_{2} \mu}$ of $S L(2) \otimes S L(n)$ at $n_{c}=0$ and

$$
\begin{array}{cccc}
n_{c}=1 & n_{c}=2 & n_{c}=3 & n_{c}=4 \\
P_{s l_{2} \otimes s l_{n} \mu \alpha i j} & P_{s l_{2} \otimes s l_{n} \mu i_{1} \ldots i_{4}} & P_{\mu \alpha, i_{1} \ldots i_{6}} & P_{s l_{2} \otimes s l_{n} \mu j_{1} \ldots j_{7} i}
\end{array} .
$$

The Cartan form contains the factor $e^{\frac{1}{\sqrt{2}} \vec{\phi} \cdot \vec{\alpha}}$, contained in the $g^{-1}$ part of $g^{-1} \partial_{\mu} g$, and so using equation (2.43) we find that the level $n_{c}$ contribution comes with the factor

$$
e^{\frac{1}{\sqrt{2}} n_{c} x \rho}=e^{2 \alpha \rho n_{c} \frac{8-n}{n}} .
$$

The ten dimensional origin of the first two terms of equation (2.44) is obvious given their index structure and they are contained in the blocks $\mathcal{F}_{s l_{2} \otimes s l_{n} \alpha \mu i j}$ and $F_{s l_{2} \otimes s l_{n} \mu i_{1} \ldots i_{4}}$ respectively that come from the dimensional reduction of the three form and five form field strengths respectively. The fourth term of equation (2.44) only occurs for $d=3$ and $d=4$ and in these dimensions it arises as the dual of the three from field strength, more precisely the dual of $\mathcal{F}_{s l_{2} \otimes s l_{n}} \alpha \nu_{1} \nu_{2} \nu_{3}$ and $\mathcal{F}_{s l_{2} \otimes s l_{n}} \alpha \nu_{1} \nu_{2} i$ respectively. Alternatively, one can think of the fourth term as arising from the dimensional reduction of the field strength $\mathcal{F}_{s l 2 \alpha \nu_{1} \ldots \nu_{7}}$. The final term in equation (2.44) only occurs in $d=3$ dimensions, that is for $E_{8}$, and it arises as the dual of the graviphoton $\partial_{[a} h_{b]}{ }^{i}$. At the end of section three we showed that calculating the powers $e^{\rho}$ from the original field, or its dual, gave the same result. As such we will calculate it from the Cartan forms of equation (2.45). We observe that these carry one $d$ dimensional spacetime index and $2 n_{c}$ internal indices and according to the discussion around equation (2.24) we find a factor of

$$
e^{-\rho\left(\alpha+2 n_{c} \beta\right)}=e^{2 \alpha \rho n_{c} \frac{8-n}{n}} e^{-\alpha \rho}
$$

for each contribution.

Thus for each factor of the Cartan form $P_{E \mu}$ in the $d$ dimensional effective action we find an additional factor of $e^{-\alpha \rho}$ in the dimensionally reduced action compared to the $E_{n+1}$ formulation. This result, taken together with the previous result for factors of the Riemann tensor, is consistent with the rule that for each spacetime derivative in $d$ dimensions we get an additional factor of $e^{-\alpha \rho}$.

To treat the other building blocks in the same way we must learn how to decompose more general representations of $E_{n+1}$ into those of $S L(2) \otimes S L(n)$. To do this we use the technique of reference $[44,45]$. If one wants to consider the fundamental representation $\vec{\lambda}^{i}$ of $E_{n+1}$ associated with the node labeled $i$ we add a new node, denoted $\star$, to the $E_{n+1}$ 
Dynkin diagram which is connected to the node labeled $i$ by a single line to construct the Dynkin diagram for an enlarged algebra of rank $n+2$. Deleting the $\star$-node we recover the $E_{n+1}$ Dynkin diagram and the $\vec{\lambda}^{i}$ of $E_{n+1}$ is found in the adjoint representation of the enlarged algebra provided we keep only contributions at level $n_{\star}=1$. Thus we find the decomposition of the fundamental representation of $E_{n+1}$ into representations of $S L(2) \otimes$ $S L(n)$ by decomposing the adjoint representation of the enlarged algebra but deleting the additional node and keeping only contributions with $n_{\star}=1$ and deleting node $n$ but keeping all levels of $n_{c}$. The level one states are a representation as the commutator preserves the level and so the commutator of the level zero generators, that is the adjoint representation of $E_{n+1}$, with the level one states give again level one states. It is the desired representation since the lowest state contains $\underline{\lambda}^{i}$. For the details see reference [44,45].

The weights of the $\vec{\lambda}^{i}$ representation of $E_{n+1}$ can be written in the form

$$
\left([\mu], n_{c} x-\frac{1}{x} \underline{\lambda}^{n-2} \cdot \underline{\lambda}^{i},[\underline{]}]\right),
$$

except for $i=n$ for which it is of the form $\left([\mu], n_{c} x-\frac{1}{x},[\underline{\lambda}]\right)$ Here $(\mu, \underline{\lambda})$ is the highest weight of the $S L(2) \otimes S L(n)$ representation that occurs. We note that $\vec{\nu} \cdot \vec{\lambda}^{i}=\frac{2 i}{n}$ for $i \leq n-2, \vec{\nu} \cdot \vec{\lambda}^{n-1}=\frac{(n-2)}{n}$ and $\vec{\nu} \cdot \vec{\lambda}^{n+1}=\frac{1}{2}$.

Next we will treat the two form field strengths in the $d$ dimensional effective action in a similar way. The one form gauge field, from which they are constructed, belong to the $\vec{\lambda}^{1}$ representation of $E_{n+1}$. The $\vec{\lambda}^{1}$ representation of $E_{n+1}$ decomposes into $S L(2) \otimes S L(n)$ representations as follows

$$
\begin{array}{ccccccc}
n_{c}=0 & n_{c}=1 & n_{c}=2 & n_{c}=3 & n_{c}=4 & n_{c}=4 & n_{c}=4 \\
\left(0, \underline{\lambda}^{1}\right) & \left(\mu, \underline{\lambda}^{n-1}\right) & \left(0, \underline{\lambda}^{n-3}\right) & \left(\mu, \underline{\lambda}^{n-5}\right) & \left(2 \mu, \underline{\lambda}^{n-7}\right) & \left(\mu, \underline{\lambda}^{n-7}\right) & \left(0, \underline{\lambda}^{n-1}+\underline{\lambda}^{n-6}\right) \\
n_{c}=5 & n_{c}=6 & n_{c}=7 & n_{c}=8 \\
\left(\mu, \underline{\lambda}^{n-2}+\underline{\lambda}^{n-7}\right) & \left(0, \underline{\lambda}^{n-4}+\underline{\lambda}^{n-7}\right) & \left(\mu, \underline{\lambda}^{n-6}+\underline{\lambda}^{n-7}\right) & \left(0, \underline{\lambda}^{n-1}+2 \underline{\lambda}^{n-7}\right) .
\end{array}
$$

The reader may verify that one finds the correct dimensions of the $\vec{\lambda}^{1}$ representation, that is $16,27,56$ and 248 for $n=4,5,6$ and 7 . The weights of the $\vec{\lambda}^{1}$ representation are given by

$$
\begin{gathered}
\left(0, \frac{2}{n x},\left[\underline{\lambda}^{1}\right]\right),\left(\left[\mu_{1}\right], \frac{2}{n x}-x,\left[\underline{\lambda}^{n-1}\right]\right),\left(0, \frac{2}{n x}-2 x,\left[\underline{\lambda}^{n-3}\right]\right),\left(\left[\mu_{1}\right], \frac{2}{n x}-3 x,\left[\underline{\lambda}^{n-5}\right]\right), \\
\left(2\left[\mu_{1}\right], \frac{1}{2 x}-4 x,\left[\underline{\lambda}^{n-7}\right]\right),\left(0, \frac{2}{n x}-4 x,\left[\underline{\lambda}^{n-7}\right]\right),\left(0, \frac{2}{n x}-4 x,\left[\underline{\lambda}^{1}\right]+\left[\underline{\lambda}^{n-6}\right]\right) \\
\left(\left[\mu_{1}\right], \frac{2}{n x}-5 x,\left[\underline{\lambda}^{n-2}+\underline{\lambda}^{n-7}\right]\right),\left(0, \frac{2}{n x}-6 x,\left[\underline{\lambda}^{n-4}+\underline{\lambda}^{n-7}\right]\right) \\
\left(\left[\mu_{1}\right], \frac{2}{n x}-7 x,\left[\underline{\lambda}^{n-6}+\underline{\lambda}^{n-7}\right]\right),\left(\left[\mu_{1}\right], \frac{2}{n x}-8 x,\left[\underline{\lambda}^{1}+2 \underline{\lambda}^{n-7}\right]\right)
\end{gathered}
$$


These correspond to two form field strengths take the form

$$
\begin{array}{ccccccc}
n_{c}=0 & n_{c}=1 & n_{c}=2 & n_{c}=3 & n_{c}=4 & n_{c}=4 & n_{c}=4 \\
\mathcal{F}_{\mu_{1} a_{2}}^{i} & \mathcal{F}_{\alpha \mu_{1} a_{2} i} & \mathcal{F}_{\mu_{1} a_{2} i_{1} i_{2} i_{3}} & \mathcal{F}_{\alpha \mu_{1} a_{2} i_{1} \ldots i_{5}} & \mathcal{F}_{\mu_{1} a_{2} j, i_{1} \ldots i_{6}} & \mathcal{F}_{\mu_{1} a_{2}(\alpha \beta), i_{1} \ldots i_{7}} & \mathcal{F}_{\mu_{1} a_{2} i_{1} \ldots i_{7}}
\end{array},
$$

as well as higher level contributions. Since a two form field strength is dual to a one form field strength in three dimensions we only study two form field strengths in dimensions four and above. This corresponds to $n \leq 6$ and so of the above field strengths we only need those at levels $n_{c}=3$ and the first term in the above equation at level $n_{c}=4$.

We recognise the two form field strengths of equation (2.51) as the dimensional reduction of the metric, i.e. the graviphoton, the three form, the five form for the first three entries. The fourth entry arises from the dual of the three form in $d=4$ and $d=5$ while the only required level four field strength is the dual of the graviphoton.

Decomposing the rank two field strength in their $E_{n+1}$ representation, using equations (2.29) and (2.32), we find the factor

$$
e^{\frac{1}{\sqrt{2}}\left(\frac{\vec{\nu} \cdot \vec{\lambda}^{1}}{x}-n_{c} x\right) \rho}=e^{\frac{2 \sqrt{2} \alpha}{n}\left(-4+n_{c}(8-n)\right) \rho},
$$

for each rank two field strength at level $n_{c}$. We observe that the above field strengths have two $d$-dimensional spacetime indices and $2 n_{c}-1$ internal indices and so the factor of $e^{\rho}$ that appears when carrying out the dimensional reduction from ten dimensions is

$$
e^{-\rho\left(2 \alpha+\left(2 n_{c}-1\right) \beta\right)}=e^{\frac{2 \sqrt{2} \alpha}{n}\left(-4+n_{c}(8-n)\right) \rho} e^{-\alpha \rho} .
$$

Evaluating this and comparing with the factor in equation (2.52) we find an additional factor of $e^{-\alpha \rho}$ for each rank two field strength.

We now carry out the same analysis for the rank three field strengths. We need only consider these field strengths in dimensions $d \geq 6$, since in a lower dimension a rank three field strength is dual to a lower rank field strength. This is equivalent to $n \leq 4$. The rank three field strength belong to the $\vec{\lambda}^{n+1}$ representation of $E_{n+1}$. One finds that the weights in the $\vec{\lambda}^{n+1}$ representation of $E_{n+1}$ have the form

$$
\begin{gathered}
\left(\left[\mu_{1}\right], \frac{1}{2 x}, \underline{0}\right),\left(0, \frac{1}{2 x}-x,\left[\underline{\lambda}^{n-2}\right]\right),\left(\left[\mu_{1}\right], \frac{1}{2 x}-2 x,\left[\underline{\lambda}^{n-7}\right]\right), \\
\left(0, \frac{1}{2 x}-3 x,\left[\underline{\lambda}^{n-1}\right]+\left[\underline{\lambda}^{n-5}\right]\right),\left(0, \frac{1}{2 x}-3 x,\left[\underline{\lambda}^{n-6}\right]\right),\left(\left[\beta_{1}\right], \frac{1}{2 x}-3 x,\left[\underline{\lambda}^{n-6}\right]\right), \\
\left(\left[\mu_{1}\right], \frac{1}{2 x}-4 x,\left[\underline{\lambda}^{n-1}+\underline{\lambda}^{n-7}\right]\right),\left(\left[\mu_{1}\right], \frac{1}{2 x}-4 x,\left[\underline{\lambda}^{n-6}+\underline{\lambda}^{n-2}\right]\right), \\
\left(0, \frac{1}{2 x}-5 x,\left[\underline{\lambda}^{n-4}+\underline{\lambda}^{n-6}\right]\right),\left(0, \frac{1}{2 x}-5 x,\left[\underline{\lambda}^{n-1}+\underline{\lambda}^{n-2}+\underline{\lambda}^{n-7}\right]\right),
\end{gathered}
$$




$$
\begin{gathered}
\left(\left[\beta_{1}\right], \frac{1}{2 x}-5 x,\left[\underline{\lambda}^{n-3}+\underline{\lambda}^{n-7}\right]\right), \\
\left(\left[\mu_{1}\right], \frac{1}{2 x}-6 x,\left[\underline{\lambda}^{n-5}+\underline{\lambda}^{n-7}\right]\right),\left(\left[\mu_{1}\right], \frac{1}{2 x}-6 x,\left[\underline{2} \lambda^{n-6}\right]\right), \\
\left(\left[\mu_{1}\right], \frac{1}{2 x}-6 x,\left[\underline{\lambda}^{n-1}+\underline{\lambda}^{n-4}+\underline{\lambda}^{n-4}\right]\right), \ldots
\end{gathered}
$$

The reader may like to verify that one has the correct count of states for the $5,10,27$, and 133-dimensional representations of $S L(5), S O(5,5), E_{6}$ and $E_{7}$ respectively. For the first few entries many contributions vanish as one has too many antisymmetrised indices. To find the 3875 dimensional representation of $E_{8}$ one must go further in the analysis.

The factor of $e^{\rho}$ associated with the term at $n_{c}$ is

$$
e^{-\frac{1}{\sqrt{2}}\left(\frac{\vec{\nu} \cdot \vec{\lambda}^{n+1}}{x}-n_{c} x\right) \rho}=e^{-\frac{2 \alpha}{n}\left(n-2 n_{c}(8-n)\right) \rho} .
$$

The corresponding field strengths carry three $d$ dimensional spacetime indices and $2 n_{c}$ internal indices and so we find in the dimensionally reduced theory a factor of

$$
e^{-\rho\left(3 \alpha+\left(2 n_{c}\right) \beta\right)}=e^{-\frac{\alpha \rho}{n}\left(3 n-2 n_{c}(8-n)\right)} .
$$

Consequently for every rank three field strength we find an additional factor of $e^{-\alpha \rho}$ in the dimensionally reduced theory. The same conclusion holds for the rank four field strengths.

Since one finds the same additional factor no matter what field strength one considers the above can be summarised as follows, for every derivative we find an additional factor of $e^{-\alpha \rho}$ in the dimensionally reduced theory. One also finds in the dimensionally reduced theory a $e^{-\hat{s} \phi}$ coming from the ten dimensional $S L(2)$ automorphic form. Consequently, the excess in the dimensionally reduced theory compared to that found in the $E_{n+1}$ formulation of equation (2.55), but not taking into account the contribution of the $E_{n+1}$ automorphic form in $d$ dimensions in the latter formulation, is given by

$$
e^{-\left(l_{T}-2\right) \alpha \rho-\hat{s} \phi}
$$

where $l_{T}=\hat{l}_{R}+\hat{l}_{1}+\hat{l}_{1}^{\prime}+\hat{l}_{3}^{+}+\hat{l}_{3}^{-}+\hat{l}_{5}$. The -2 part arises from the det $\hat{e}$. This excess can only come from the $E_{n+1}$ automorphic form. Demanding that all the weights arising from dimensional reduction of the ten dimensional theory appear in the $E_{n+1}$ formulation in $d$ dimensions we conclude that

$$
\vec{\lambda}_{\Phi}=\left(\frac{\hat{s}}{\sqrt{2}}, \alpha \frac{\left(l_{T}-2\right)}{\sqrt{2}}, \underline{0}\right)=\left(\frac{l_{T}-2}{4}+\left(l_{R R}-2 g\right)\right) \vec{\lambda}^{n+1}+\left(\frac{2 g-l_{R R}}{2}\right) \vec{\lambda}^{n}
$$

where $l_{R R}=\hat{l}_{1}^{\prime}+\hat{l}_{3}^{\prime}+\hat{l}_{5}$ counts the number of RR fields. 
Let us consider higher derivative terms constructed only out of NS-NS fields, so that $l_{R R}=0$. Suppose also that we look at terms which have a tree level, $g=0$, contribution in ten-dimensions. In this case we find the automorphic form in $d$ dimensions has the leading order behaviour $\Phi_{E} \sim e^{-\sqrt{2} \frac{\left(l_{T}-2\right)}{4} \vec{\lambda}^{n+1}}$. This strongly suggests that it is built from the $E_{n+1}$ representation with highest weight $\vec{\lambda}^{n+1}$. This is the representation that the string charges of the $d$ dimensional theory belong to.

The $S L(2, \mathbf{Z})$ Eisenstein automorphic form in ten dimensions contains two perturbative terms with dilaton dependence $e^{-s \phi}$ and $e^{(s-1) \phi}$. If the first term possesses a value of $s$ that leads to a tree level contribution then the second term leads to a genus $g=s-1 / 2$ contribution. Above we considered the effect of dimensionally reducing the tree level contribution, but one can also consider the second contribution. One finds, substituting $g=s-1 / 2$ into $(2.58)$, that the weight vector is

$$
\vec{\lambda}_{\Phi}=(1-s) \vec{\lambda}^{n+1}+(s-1 / 2) \vec{\lambda}^{n}=s \vec{\lambda}^{n+1}-(s-1 / 2) \vec{\alpha}_{n+1}
$$

However the first two terms in the perturbative contribution of the Eisenstein-like $E_{n+1}$

automorphic form in $d$ dimensions constructed using the $\vec{\lambda}^{n+1}$ representation are of the generic form $[30]$

$$
\Phi_{E} \sim E_{1} e^{-\sqrt{2} s \lambda^{n+1}}+E_{2} e^{-\sqrt{2}\left(s \vec{\lambda}^{n+1}-(s-1 / 2) \vec{\alpha}_{n+1}\right)}
$$

where $E_{1}$ and $E_{2}$ are constants. It is pleasing to see that the second term of the automorphic form in ten dimensions leads to the correct second term in the $E_{n+1}$ automorphic form in $d$ dimensions.

We note that a similar calculation for dimensional reduction of type IIA string theory on an $n$-torus leads to the same results as the the type IIB reduction considered here [46].

\section{M-Theory}

Let us now perform a similar analysis for the dimensional reduction of higher derivative terms of M-theory. Note that to compare with the previous section one must make the substitution $n \rightarrow n+1$. In addition the values of $\alpha$ and $x$ in this section are different to those of section 2 .

The Bosonic field content of M-theory consists of the graviton with curvature $\hat{R}$ and a three form gauge field $\hat{A}_{\hat{\mu} \hat{\nu} \hat{\rho}}$ out of which the four form field strength $\hat{F}_{\hat{\mu} \hat{\nu} \hat{\rho} \hat{\sigma}}$ is constructed. At lowest order in derivatives the low energy effective action may be written

$$
\int d^{11} x \operatorname{det} \hat{e}\left(\hat{R}-\frac{1}{2 \cdot 4 !} \hat{F}^{2}+\ldots\right)
$$


where the ellipsis denote Fermion terms as well as a Chern-Simons-type term for $\hat{A}$. A generic higher derivative correction in the $d=11$ low energy effective action of M-theory may be written,

$$
\int d^{11} x \operatorname{det} \hat{e}(\partial)^{\hat{l}_{0}} \hat{R}^{\frac{\hat{l}_{1}}{2}} \hat{F}^{\hat{l}_{4}}
$$

M-theory, dimensionally reduced on an $n$-torus, possesses an $E_{n}$ symmetry in $d=11-$ $n$ dimensions and shares the same manifest $S L(n)$ symmetry through the non-linearly realised field strengths and the Cartan forms in $d$ dimensions as the type IIB theory. However, no dilatonic scalar is present in $d=11$ dimensions. Upon dimensional reduction, a higher derivative term will pick up a dependence on the $n$ diagonal components of the metric on the $n$-torus $\rho$ and $\underline{\phi}$. We observe that the higher derivative terms in the dimensionally reduced formulation carry a factor of $e^{\sqrt{2} \vec{w} \cdot \vec{\phi}}$ where the $n$ vectors $\vec{w}$ and $\vec{\phi}$ recording the dilatonic scalar field content and their associated weights are defined as

$$
\begin{aligned}
\vec{\phi} & =(\rho, \underline{\phi}), \\
\vec{w} & =(\kappa, \underline{w}) .
\end{aligned}
$$

The general term in the $E_{n}$ formulation in $d$ dimensions is a polynomial in the non-linearly realised field strengths $\mathcal{F}$, Cartan forms $P$ and curvature $R$ multiplied by an automorphic form $\Phi_{E}$ constructed out of some representation of $E_{n}$

$$
\int d^{d} x \operatorname{det} e \partial^{l_{0}} R^{\frac{l_{R}}{2}} P_{E \mu_{1}}^{l_{1}}\left(\mathcal{F}_{E \mu_{1} \mu_{2}}\right)^{l_{2}}\left(\mathcal{F}_{E \mu_{1} \mu_{2} \mu_{3}}\right)^{l_{3}} \Phi_{E} \ldots
$$

We will again determine the representation out of which the $E_{n}$ automorphic form is constructed in $d$ dimensions by comparing the dimensionally reduced formulation, with manifest $S L(n)$ symmetry, to that of the $E_{n}$ formulation. The Dynkin diagram for Mtheory is (note that here we use a different labeling for the nodes and hence the roots and weights are also labeled differently than in section 2)

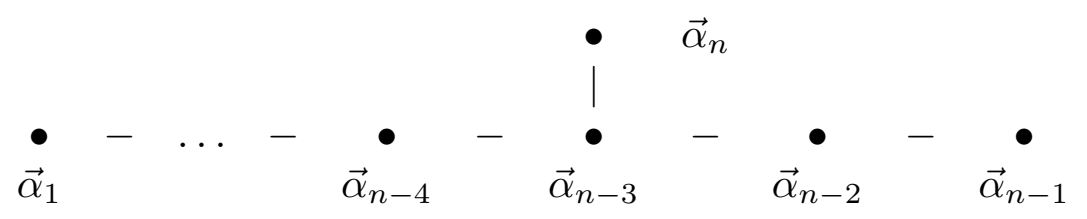

Fig. 3 Dynkin diagram for $E_{n+1}$ in M-theory labelling

The simple roots of $E_{n}$ may be written as

$$
\vec{\alpha}_{i}=\left(0, \underline{\alpha}_{i}\right), \quad i=1, \ldots, n-1, \quad \vec{\alpha}_{n}=(x, \underline{0})-\vec{\mu},
$$


where $\vec{\mu}=\left(0, \underline{\lambda}^{n-3}\right)$. The variable $x$ associated with the $\rho$ factor of the deleted node $n$ is evaluated via the inner products between the simple roots of $E_{n}$, given by the corresponding Cartan matrix, one finds

$$
x=\sqrt{\frac{9-n}{n}}=(3 \sqrt{2} \alpha)^{-1} .
$$

The fundamental weights of $E_{n}$, dual to the simple roots $\underline{\alpha}_{i}$, are

$$
\begin{aligned}
\vec{\lambda}^{i} & =\left(\frac{1}{x} \underline{\lambda}^{i} \cdot \underline{\lambda}^{n-3}, \underline{\lambda}^{i}\right), \\
\vec{\lambda}^{n} & =\left(\frac{1}{x}, \underline{0}\right) .
\end{aligned}
$$

One may write any root of $E_{n}$ as

$$
\vec{\alpha}=n_{c} \vec{\alpha}_{n}+\sum_{i=1}^{n-1} n_{i} \vec{\alpha}_{i}=n_{c}(x, \underline{0})-\vec{\lambda}
$$

where $\vec{\lambda}=n_{c} \vec{\nu}-\sum_{i=1}^{n-1} n_{i} \vec{\alpha}_{i}$. As in the IIB theory, if a representation of $S L(n)$ is present at some level $n_{c}$ in the adjoint representation of $E_{n}$, then its highest weight may be written as $\vec{\lambda}$ for some combination of the integers $n_{c}$ and $n_{i}$. Level $n_{c}=0$ contains the adjoint representation of $S L(n)$. The highest weight representations of $S L(n)$ at higher levels are

$$
\begin{array}{ccc}
n_{c}=1 & n_{c}=2 & n_{c}=3 \\
\underline{\lambda}^{3} & \underline{\lambda}^{6} & \underline{\lambda}^{1}+\underline{\lambda}^{n-8}
\end{array}
$$

So the weights in the lower levels of the adjoint representation of $E_{n}$ are

$$
\left(0,\left[\underline{\alpha}_{1}+\ldots+\underline{\alpha}_{n-1}\right]\right),\left(x,\left[\underline{\lambda}^{3}\right]\right),\left(2 x,\left[\underline{\lambda}^{6}\right]\right),\left(3 x,\left[\underline{\lambda}^{1}+\underline{\lambda}^{n-8}\right]\right)
$$

The decomposition of the Cartan form $P_{E}$, at a given level $n_{c}$ is found by examining the weights. At level $n_{c}=0$ the Cartan form $P_{E}$ contains the Cartan form of $S L(n)$ at higher levels the Cartan form $P_{E}$ decomposes as follows

$$
\begin{array}{ccc}
n_{c}=1 & n_{c}=2 & n_{c}=3 \\
P_{s l_{n} i_{1} i_{2} i_{3}} & P_{s l_{n} i_{1} \ldots i_{6}} & P_{s l_{n} j, i_{1} \ldots i_{8}}
\end{array} .
$$

The Cartan form contains the factor $e^{\frac{1}{\sqrt{2}} \vec{\phi} \cdot \vec{\alpha}}$, so at level $n_{c}$ we find a factor of

$$
e^{\frac{1}{\sqrt{2}}\left(\left(n_{c} x\right) \rho\right)}=e^{\left(3 n_{c}\right) \alpha \rho\left(\frac{9-n}{n}\right)}
$$

With the natural ordering on the levels, we find the maximum level that contributes is $n_{c}=1$ for $n=3,4, n_{c}=2$ for $n=5,6$ and $n_{c}=3$ for $n=7$. The $S L(n)$ Cartan forms 
$P_{S L(n)}$ originate from the four form field strength $\hat{G}_{\mu \bar{i}_{1} \bar{i}_{2} \bar{i}_{3}}$ at level $n_{c}=1$, the dual of the four form field strength at level $n_{c}=2$ and the graviphoton at level $n_{c}=3$. These Cartan forms of $S L(n)$, arising upon dimensional reduction, carry one $d$ dimensional spacetime index and $\left(3 n_{c}\right)$ internal indices. Therefore, each Cartan form of $S L(n)$, at level $n_{c}$, is multiplied by the factor

$$
e^{-\rho\left(\alpha+\left(3 n_{c}\right) \beta\right)}=e^{\left(3 n_{c}\right) \alpha \rho\left(\frac{9-n}{n}\right)} e^{-\alpha \rho} .
$$

The two form field strengths lie in the representation of $E_{n}$ with highest weight $\vec{\lambda}^{1}$. Decomposing the $\vec{\lambda}^{1}$ of $E_{n}$ into representations of $S L(n)$ level by level, we find

$$
\begin{array}{cccc}
n_{c}=0 & n_{c}=1 & n_{c}=2 & n_{c}=3 \\
\underline{\lambda}^{1} & \underline{\lambda}^{n-2} & \underline{\lambda}^{n-5} & \underline{\lambda}^{n-1}+\underline{\lambda}^{n-7} .
\end{array}
$$

Therefore, for $n \leq 7$, the weights of the $\vec{\lambda}^{1}$ representation are

$$
\left(\frac{3}{n x},\left[\underline{\lambda}^{1}\right]\right),\left(\frac{3}{n x}-x,\left[\underline{\lambda}^{n-2}\right]\right),\left(\frac{3}{n x}-2 x,\left[\underline{\lambda}^{n-5}\right]\right),\left(\frac{3}{n x}-3 x,\left[\underline{\lambda}^{n-1}+\underline{\lambda}^{n-7}\right]\right) .
$$

From the weights, we see that the corresponding two form field strengths, at each level, are

$$
\begin{array}{cccc}
n_{c}=0 & n_{c}=1 & n_{c}=2 & n_{c}=3 \\
\mathcal{F}_{\mu_{1} \mu_{2}}^{i} & \mathcal{F}_{\mu_{1} \mu_{2} i_{1} i_{2}} & \mathcal{F}_{\mu_{1} \mu_{2} i_{1} \ldots i_{5}} & \mathcal{F}_{\mu_{1} \mu_{2} i, j_{1} \ldots j_{7}} .
\end{array}
$$

The two form field strengths appear in $d \geq 4$ dimensions. In $d=11-n$ dimensions one finds that all two form field strengths, with associated level $n_{c}$, satisfying the constraint $n \leq 3 n_{c}-1$ will be present. We see that the two form field strength at level $n_{c}=0$ arises through the dimensional reduction of the metric and four form at levels $n_{c}=0, n_{c}=1$ respectively. The two remaining levels in the decomposition of the $\vec{\lambda}^{1}$ are associated with the duals of the four form field strength and the graviphoton at $n_{c}=2$ and $n_{c}=3$ respectively. Since the two form field strengths in the $E_{n}$ lie in some representation of $S L(n)$ at level $n_{c}$ in the decomposition of $\vec{\lambda}^{1}$ they carry a multiplicative factor of

$$
e^{\frac{1}{\sqrt{2}}\left(\left(-\frac{3}{n x}+n_{c} x\right) \rho\right)}=e^{-\frac{9}{n}-n_{c} \alpha \rho\left(\frac{9-n}{n}\right)} .
$$

If we compare the multiplicative factor found through the decomposition of the $\vec{\lambda}^{1}$ in the $E_{n}$ formulation to the corresponding factor arising in the dimensionally reduced formulation, where the two form field strengths carry two $d$ dimensional indices and $3 n_{c}-1$ internal indices, and so appear multiplied by the factor

$$
e^{-\rho\left(2 \alpha+\left(3 n_{c}-1\right) \beta\right)}=e^{-\alpha \rho} e^{-\frac{9}{n}-n_{c} \alpha \rho\left(\frac{9-n}{n}\right)},
$$

we find that the two form field strengths in the dimensionally reduced M-theory formulation carry a surplus factor of $e^{-\alpha \rho}$. In the $E_{n}$ formulation the three form field strengths lie in 
the representation with highest weight $\vec{\lambda}^{n-1}$. One finds that the $\vec{\lambda}^{n+1}$ representation of $E_{n}$ decomposes, in the following way for $n \leq 5$

$$
\begin{array}{cc}
n_{c}=0 & n_{c}=1 \\
\underline{\lambda}^{n-1} & \underline{\lambda}^{n-4} .
\end{array}
$$

We observe that, for $n \leq 5$, the weights in the $\vec{\lambda}^{n-1}$ representation of $E_{n}$ are

$$
\left(\left(\frac{n-3}{n x}\right),\left[\underline{\lambda}^{n-1}\right]\right),\left(\frac{(n-3)}{n x}-x,\left[\underline{\lambda}^{n-4}\right]\right) .
$$

The three form field strengths, at level $n_{c}$, are

$$
\begin{array}{cc}
n_{c}=0 & n_{c}=1 \\
\mathcal{F}_{\mu_{1} \mu_{2} \mu_{3} i} & \mathcal{F}_{\mu_{1} \mu_{2} \mu_{3} i_{1} \ldots i_{4}} .
\end{array}
$$

The three form field strength occurring in the decomposition of the $\lambda^{n-1}$ at level $n_{c}=0$ arises from the dimensional reduction of the four form field strength, while the other, at level $n_{c}=1$ is associated with the dual of the dimensionally reduced four form field strength. The three form field strengths at levels $n_{c}=0,1$ appear in $d=6,7$ dimensions, in $d=8$ only the $n_{c}=0$ three form field strength is present. The decomposition of the $\vec{\lambda}^{n-1}$ of $E_{n}$, at level $n_{c}$, is multiplied by a factor of

$$
e^{-\frac{1}{\sqrt{2}}\left(\frac{(n-3)}{n x}-n_{c} x\right) \rho}=e^{\left(\left(-3+\frac{9}{n}\right)+n_{c}\left(\frac{9-n}{n}\right)\right) \alpha \rho} .
$$

The three form field strengths in the dimensionally reduced formulation come with three spacetime indices and $\left(3 n_{c}+1\right)$ internal indices, therefore they carry a factor of

$$
e^{-\rho\left(3 \alpha+\left(3 n_{c}+1\right) \beta\right)}=e^{-\alpha \rho} e^{\left(\left(-3+\frac{9}{n}\right)+n_{c}\left(\frac{9-n}{n}\right)\right) \alpha \rho} .
$$

In $d=11-n$ dimensions, the Cartan forms, field strengths and curvatures lying in the $E_{n}$ representation may be constructed out of the dimensionally reduced Cartan forms, field strengths and curvatures with manifest $S L(n)$ symmetry. For example, the two form field strengths in $d=7$ dimensions lie in the $\mathbf{1 0}$ of $E_{4}$, which may be constructed out of the two form field strengths arising from dimensional reduction to $d=7$. Namely, the graviphotons lying in the 4 and the dimensionally reduced four form field strength $\hat{G}_{\mu_{1} \mu_{2} \bar{i}_{1} \bar{i}_{2}}$ lying in the $\underline{\mathbf{6}}$ of $S L(4)$. However, each of the dimensionally reduced terms carry an additional factor of $e^{-\alpha \rho}$. Therefore, any product of Cartan forms, field strengths and curvatures, in the $E_{n}$ formulation, reconstructed using the appropriate dimensionally reduced terms, will be multiplied by a surplus factor of

$$
e^{-\left(l_{T}-2\right) \alpha \rho}
$$


where $l_{T}$ is the total number of derivatives in the product. This factor must be attributed to the automorphic form in the $E_{n}$ formulation. To leading order, we may write the automorphic form in the $E_{n}$ formulation as $\Phi_{E_{n}} \sim e^{-\sqrt{2} \vec{\lambda}_{\Phi} \cdot \vec{\phi}}$. Thus, one finds

$$
\vec{\lambda}_{\Phi}=\left(\alpha\left(\frac{l^{T}-2}{\sqrt{2}}\right), \underline{0}\right)=\left(\frac{l_{T}-2}{6}\right) \vec{\lambda}^{n} .
$$

\section{Discussion}

In this paper we have dimensionally reduced the higher derivative terms of ten dimensional IIB theory and deduced the weight vectors that are associated with the Cartan subalgebra fields of the $E_{n+1}$ symmetry. Most of these weights are accounted for once the $d$-dimensional theory is expressed in terms of $E_{n+1}$ covariant building blocks involving the Riemann tensor, field strengths and derivatives of the scalars. However, we also found that there was always a remaining weight. This implies that polynomials constructed only out of the field strengths are not consistent with U-duality in the lower dimension. On the other hand these additional weights can be accounted for in the $d$ dimensional theory if they are attributed to an $E_{n+1}$ automorphic form. In this way we obtained constraints on the automorphic forms that occur in $d$-dimensions.

Carrying out this procedure we have found that the dimensional reduction of the IIB higher derivative corrections implies that such terms in $d$ dimensions should contain an automorphic form involving the weight $\vec{\lambda}^{n+1}$, using the labeling of the Dynkin diagram of Figure 1. It is natural to think of this as the highest weight of the representation used to construct the automorphic form. This applies to all terms in a given dimension, although this does not mean that the same automorphic appears for all terms. For terms that only contain the Riemann tensor and scalars the leading order weight can be readily deduced by counting the number of inverse metrics required, however for more general terms we needed to perform a detailed group theory analysis.

As the constraints we find arise from considering the ten dimensional theory we are in effect considering terms that survive the decompactification from $d$ dimensions, that is $\rho \rightarrow-\infty$. We have focused particularly on the terms that arise at tree level in ten dimensions. However we also saw that the next-to-leading order contribution in ten-dimensions correctly matched that of the $d$-dimensional automorphic form if the $\vec{\lambda}^{n+1}$ representation is used for the case of Einstein-like automorphic forms.

This result is in agreement with the results [17-36] found so far for terms with low numbers of spacetime derivatives in that the automorphic forms studied for these terms are constructed from the $\vec{\lambda}^{n+1}$ representation. It is also natural in that the string charges belong to the $\vec{\lambda}^{n+1}$ multiplet and the discrete $E_{n+1}$ group acts naturally on these objects. 
We also performed a similar calculation from the viewpoint of eleven-dimensional Mtheory. We found that the automorphic forms should contain the weight $\vec{\lambda}^{n-1}$, using the type IIB labeling of the $E_{n+1}$ Dynkin diagram of Figure 1. This is also natural as membrane charges belong to the $\vec{\lambda}^{n-1}$ representation. It would be interesting to reconcile this result with that from the IIB perspective. The automorphic forms contain combinations of weights and one would have to find the combination of weights predicted from the Mtheory viewpoint in the automorphic from constructed from the representation with highest weight $\vec{\lambda}^{n+1}$ that it used in the type IIB theory. In this way the M-theory analysis places a non-trivial constraint on the automorphic forms.

A more radical possibility is that the automorphic forms for these different representations and suitable $s$ are actually the same. In fact for $S L(5)$ the two representations

are the $\mathbf{5}$ of the string and the $\overline{\mathbf{5}}$ of the membrane, the highest weight of the former being minus the lowest weight of the latter, lead to automorphic forms that are indeed related for suitable values of $s$. Indeed, for the higher rank groups some correspondences of this type for the automorphic forms corresponding to terms with low numbers of spacetime derivatives have already have been conjectured in $[27,36]$. This would require very considerable conspiracies since the representations involved are quite different (including vastly different dimensions).

Another possibility is that a given higher derivative term can involve more than one automorphic form based on different representations. This has already been found to occur in seven dimensions for the $\partial^{4} R^{4}$ term [35].

\section{Acknowledgments}

Peter West would like to thank The Erwin Schroedinger International Institute for Mathematical Physics and the Theoretical Physics Department of the Vienna University of technology for their kind hospitality in October 2009 when some of the work in this paper was carried out. This work has been supported by an STFC Rolling grant ST/G000395/1.

\section{Appendix A: Non-linear Realisations}

In this appendix we review of the construction of non-linear realisations in a form suitable to that used in this paper. We consider a group $G$ with Lie algebra Lie $(G)$. $\operatorname{Lie}(G)$ can be split into the Cartan subalgebra with elements $\vec{H}$, positive root generators $E_{\vec{\alpha}}$ and negative root generators $E_{-\vec{\alpha}}$ with $\vec{\alpha}>0$. There exists a natural involution, known as the Cartan involution, defined by

$$
\tau:\left(\vec{H}, E_{\vec{\alpha}}\right) \rightarrow-\left(\vec{H}, E_{-\vec{\alpha}}\right) .
$$


To construct the non-linear realisation we must specify a subgroup $H$ (not to be confused with the generators of the Cartan subgroup which are denoted by $\vec{H}$ ). For us this is defined to be the subgroup left invariant under the Cartan involution, i.e. $H=\{g \in G: \tau(g)=g\}$. In terms of the Lie algebra $\operatorname{Lie}(H)$ it is all elements $A$ such that $A=\tau(A)$.

The non-linear realisation is constructed from group elements $g(x) \in G$ that depend on spacetime that are subject to the transformations

$$
g(x) \rightarrow g_{0} g(x) h^{-1}(x)
$$

where $g_{0} \in G$ is constant and $h(x) \in H$ depends on spacetime. We may write the group element in the form

$$
g(x)=e^{\sum_{\vec{\alpha}>0} \chi_{\vec{\alpha}} E_{\vec{\alpha}}} e^{-\frac{1}{\sqrt{2}} \vec{\phi} \cdot \vec{H}} e^{\sum_{\vec{\alpha}>0} u_{\vec{\alpha}} E_{-\vec{\alpha}}}
$$

but using the local transformation we can bring it to the form

$$
g(\xi)=e^{\sum_{\vec{\alpha}>0} \chi_{\vec{\alpha}} E_{\vec{\alpha}}} e^{-\frac{1}{\sqrt{2}} \vec{\phi} \cdot \vec{H}} .
$$

Here we use $\xi=\left(\vec{\phi}, \chi_{\vec{\alpha}}\right)$ as a generic symbol for all the scalar fields, which are functions of spacetime, that parameterize the coset representative. Under a rigid $g_{0} \in G$ transformation $g(\xi) \rightarrow g_{0} g(\xi)$ this form for the coset representative is not preserved. However one can make a compensating transformation $h\left(g_{0}, \xi\right) \in H$ that returns $g_{0} g(\xi)$ into the form of equation (A.5);

$$
g_{0} g(\xi) h^{-1}\left(g_{0}, \xi\right)=g\left(g_{0} \cdot \xi\right)
$$

This induces a non-linear action of the group $G$ on the scalars; $\xi \rightarrow g_{0} \cdot \xi$.

We will also need a linear representation of $G$. Let $\vec{\mu}^{i}, i=1, \ldots, N$ be the weights of the representation and $\left|\vec{\mu}^{i}\right\rangle$ be a corresponding states. We choose $\vec{\mu}^{1}$ to be the highest weight and so the corresponding state satisfies $E_{\vec{\alpha}} \mid \vec{\mu}^{1}>=0$ for all simple roots $\vec{\alpha}$. The states in the rest of the representation are polynomials of $F_{\vec{\alpha}}=E_{-\alpha}$ acting on the highest weight state.

We consider states of the form $|\psi\rangle=\sum_{i} \psi_{i}\left|\vec{\mu}^{i}\right\rangle$. Under the action $U\left(g_{0}\right)$ of the group $G$ we have

$$
\left|\psi>\rightarrow U\left(g_{0}\right)\right| \psi>=L\left(g_{0}^{-1}\right) \sum_{i} \psi_{i}\left|\vec{\mu}^{i}>\equiv\left(U\left(g_{0}\right) \psi_{i}\right)\right| \vec{\mu}^{i}>=\sum_{i, j} D_{i}^{j}\left(g_{0}^{-1}\right) \psi_{j} \mid \vec{\mu}^{i}>
$$

where $L\left(g_{0}\right)$ is the expression of the group element $g_{0}$ in terms of the Lie algebra elements which now act on the states of the representation in the usual way. We note that the action of the group on the components $\psi_{i}$ is given by $\psi_{i} \rightarrow U\left(g_{0}\right) \psi_{i}=\sum_{j} D_{i}{ }^{j}\left(g_{0}^{-1}\right) \psi_{j}$ which is the result expected for a passive action. The advantage of using the states to 
discuss the representation is that we can use the action of the Lie algebra elements $L\left(g_{0}\right)$ on the states to compute the matrix $D_{i}{ }^{j}$ of the representation and deduce properties of the representation in general.

Given any linear realisation, such as the one in equation (A.8), we can construct a non-linear realisation by

$$
\left|\varphi(\xi)>=\sum \varphi_{i}\right| \vec{\mu}^{i}>=L\left(g^{-1}(\xi)\right)\left|\psi>=e^{\sum_{\vec{\alpha}>0} e^{\frac{1}{\sqrt{2}} \vec{\phi} \cdot \vec{H}}} e^{-\sum_{\vec{\alpha}>0} \chi_{\vec{\alpha}} E_{\vec{\alpha}}}\right| \psi>
$$

where $g(\xi)$ is the group element of the non-linear realisation in equation (A.5). Under a group transformation $U\left(g_{0}\right)$ it transforms as

$$
\begin{aligned}
U\left(g_{0}\right) \mid \varphi(\xi)> & =L\left(g^{-1}(\xi)\right) U\left(g_{0}\right)\left|\psi>=L\left(g^{-1}(\xi)\right) L\left(g_{0}^{-1}\right)\right| \psi> \\
& =L\left(\left(g_{0} g^{-1}(\xi)\right) \mid \psi>\right. \\
& =L\left(h^{-1}\right) \mid \varphi\left(g_{0} \cdot \xi\right)>
\end{aligned}
$$

using equation (A.2). In terms of the component fields we find that $\varphi_{i}(\xi)=$ $\sum_{j} D_{i}{ }^{j}\left(g^{-1}(\xi)\right) \psi_{j}$ and $U\left(g_{0}\right) \varphi_{i}(\xi)=\sum_{j} D_{i}{ }^{j}\left((h)^{-1}\right) \varphi_{j}\left(g_{0} \cdot \xi\right)$. The reader can find the example of $S L(2)$ worked out in section 2.1 .

\section{References}

[1] I. C. G. Campbell and P. C. West, Nucl. Phys. B 243, 112 (1984).

[2] F. Giani and M. Pernici, Phys. Rev. D 30, 325 (1984).

[3] M. Huq and M. A. Namazie, Class. Quant. Grav. 2, 293 (1985) [Erratum-ibid. 2, 597 (1985)].

[4] J. H. Schwarz and P. C. West, Phys. Lett. B 126, 301 (1983).

[5] P. S. Howe and P. C. West, Nucl. Phys. B 238, 181 (1984).

[6] J. H. Schwarz, Nucl. Phys. B 226, 269 (1983).

[7] E. Cremmer, B. Julia and J. Scherk, Phys. Lett. B 76, 409 (1978).

[8] E. Cremmer and B. Julia, Phys. Lett. B 80, 48 (1978).

[9] N. Marcus and J. H. Schwarz, Nucl. Phys. B 228 (1983) 145.

[10] B. Julia and H. Nicolai, Nucl. Phys. B 482, 431 (1996) [arXiv:hep-th/9608082].

[11] B. Julia, in Vertex Operators and Mathematical Physics, Publications of the Mathematical Sciences Research Institute no3. Springer Verlag (1984); in Superspace and Supergravity, ed. S. W. Hawking and M. ROcek, Cambridge University Press (1981)

[12] C. Teitelboim, Phys. Lett. B167 (1986) 69.

[13] R. Nepomechie , Phys. Rev. D31, (1984) 1921;

[14] A. Sen, Nucl. Phys. B 404, 109 (1993) [arXiv:hep-th/9207053].

[15] A. Font, L. E. Ibanez, D. Lust and F. Quevedo, Phys. Lett. B 249, 35 (1990). 
[16] C. M. Hull and P. K. Townsend, Nucl. Phys. B 438, 109 (1995) [arXiv:hepth/9410167].

[17] M. B. Green and M. Gutperle, Nucl. Phys. B 498, 195 (1997) [arXiv:hep-th/9701093].

[18] M. B. Green, M. Gutperle and P. Vanhove, Phys. Lett. B 409 (1997) 177 [arXiv:hepth/9706175].

[19] M. B. Green and S. Sethi, Phys. Rev. D 59, 046006 (1999) [arXiv:hep-th/9808061].

[20] M. B. Green, H. h. Kwon and P. Vanhove, Phys. Rev. D 61, 104010 (2000) [arXiv:hepth/9910055].

[21] M. B. Green and P. Vanhove, JHEP 0601, 093 (2006) [arXiv:hep-th/0510027].

[22] M. B. Green, J. G. Russo and P. Vanhove, arXiv:hep-th/0610299.

[23] A. Basu, arXiv:hep-th/0610335.

[24] N. Berkovits and C. Vafa, Nucl. Phys. B 533, 181 (1998) [arXiv:hep-th/9803145].

[25] E. Kiritsis and B. Pioline, (p,q) string instantons," Nucl. Phys. B 508, 509 (1997) [arXiv:hep-th/9707018].

[26] A. Basu, Phys. Rev. D 77 (2008) 106003 [arXiv:0708.2950 [hep-th]].

[27] N. A. Obers and B. Pioline, Commun. Math. Phys. 209, 275 (2000) [arXiv:hepth/9903113].

[28] N. Lambert and P. West, Phys. Rev. D 74, 065002 (2006) [arXiv:hep-th/0603255].

[29] N. Lambert and P. West, Phys. Rev. D 75, 066002 (2007) [arXiv:hep-th/0611318].

[30] N. Lambert and P. West, arXiv:1001.3284 [hep-th].

[31] L. Bao, M. Cederwall and B. E. W. Nilsson, Class. Quant. Grav. 25 (2008) 095001 [arXiv:0706.1183 [hep-th]].

[32] L. Bao, J. Bielecki, M. Cederwall, B. E. W. Nilsson and D. Persson, JHEP 0807 (2008) 048 [arXiv:0710.4907 [hep-th]].

[33] L. Bao, A. Kleinschmidt, B. E. W. Nilsson, D. Persson and B. Pioline, arXiv:0909.4299 [hep-th].

[34] B. Pioline and D. Persson, arXiv:0902.3274 [hep-th].

[35] M. B. Green, J. G. Russo and P. Vanhove, arXiv:1001.2535 [hep-th].

[36] B. Pioline, arXiv:1001.3647 [hep-th].

[37] N. Berkovits, Phys. Rev. Lett. 98 (2007) 211601 [arXiv:hep-th/0609006].

[38] P. C. West, Class. Quant. Grav. 18, 4443 (2001) [arXiv:hep-th/0104081].

[39] I. Schnakenburg and P. C. West, Phys. Lett. B 517, 421 (2001) [arXiv:hepth/0107181].

[40] A. Obers and B. Pioline, Phys. Rept. 318 (1999) 113 [arXiv:hep-th/9809039].

[41] M.R Gaberdiel, D. I. Olive and P. West. Nucl. Phys. B 645 (2002) 403-437, hepth/0205068.

[42] T. Damour, M. Henneaux and H. Nicolai, Phys. Rev. Lett. 89, 221601 (2002) [arXiv:hep-th/0207267]. 
[43] P. West, Class. Quant. Grav. 20 (2003) 2393, hep-th/0307024.

[44] A. Kleinschmidt and P. West, JHEP 0402 (2004) 033, hep-th/0312247.

[45] P. West, JHEP 0408 (2004) 052, hep-th/0406150.

[46] F. Gubay and P. West, to appear. 